ITEP-TH-06/15

\title{
Yang-Baxter equations with two Planck constants
}

\author{
A. Levin ${ }^{\natural \sharp} \quad$ M. Olshanetsky $\sharp \ddagger \pitchfork \quad$ A. Zotov $\diamond \sharp 4$ \\ b - NRU HSE, Department of Mathematics, Myasnitskaya str. 20, Moscow, 101000, Russia \\ \#-ITEP, B. Cheremushkinskaya str. 25, Moscow, 117218, Russia \\ ฤ-MIPT, Inststitutskii per. 9, Dolgoprudny, Moscow region, 141700, Russia \\ $\ddagger$ - IITP (Kharkevich Institute) RAS, Bolshoy Karetny per. 19, Moscow, 127994, Russia \\ $\diamond$ - Steklov Mathematical Institute RAS, Gubkina str. 8, Moscow, 119991, Russia \\ E-mails: alevin@hse.ru, olshanet@itep.ru,zotov@mi.ras.ru
}

\begin{abstract}
We consider Yang-Baxter equations arising from its associative analog and study corresponding exchange relations. They generate finite-dimensional quantum algebras which have form of coupled GL( $N)$ Sklyanin elliptic algebras. Then we proceed to a natural generalization of the Baxter-Belavin quantum $R$-matrix to the case $\operatorname{Mat}(N, \mathbb{C})^{\otimes 2} \otimes \operatorname{Mat}(M, \mathbb{C})^{\otimes 2}$. It can be viewed as symmetric form of $\operatorname{GL}(N M) R$-matrix in the sense that the Planck constant and the spectral parameter enter (almost) symmetrically. Such type (symmetric) $R$-matrices are also shown to satisfy the Yang-Baxter like quadratic and cubic equations.
\end{abstract}




\section{Contents}

1 Introduction $\quad 2$

2 Yang-Baxter equations with two Planck constants 5

3 Quantum algebras $\quad 7$

4 Symmetric $R$-matrix in $\operatorname{Mat}(N, \mathbb{C})^{\otimes 2} \otimes \operatorname{Mat}(M, \mathbb{C})^{\otimes 2}$

5 Appendix $\quad 15$

5.1 Elliptic functions . . . . . . . . . . . . . . . . . 15

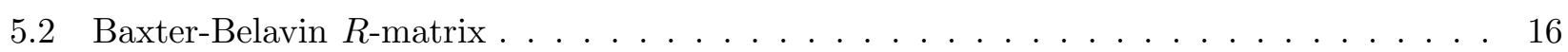

$5.3 \mathrm{GL}_{N}$ Sklyanin algebra ............................... 18

\section{Introduction}

The associative Yang-Baxter equation

$$
R_{12} R_{23}=R_{13} R_{12}+R_{23} R_{13}
$$

appeared in [1] as coassociativity condition $\left(\Delta \otimes \mathrm{id}_{A}\right) \circ \Delta=\left(\mathrm{id}_{A} \otimes \Delta\right) \circ \Delta$ for the (principle) derivation $\Delta: A \rightarrow A \otimes A$ defined in an associative algebra $A$ by $\Delta(a)=a R-R a, \forall a \in A$ and some $R \in A \otimes A$. In this paper $A=\operatorname{Mat}(N, \mathbb{C})$, i.e. $N \times N$ matrices over $\mathbb{C}$.

The equation (1.1) was extended for $R$ depending on additional parameters [18] and used for description of structures behind the classical Yang-Baxter equation on elliptic curves and their degenerations [6]. Let us write it as

$$
R_{a b}^{\hbar} R_{b c}^{\eta}=R_{a c}^{\eta} R_{a b}^{\hbar-\eta}+R_{b c}^{\eta-\hbar} R_{a c}^{\hbar}, \quad R_{a b}^{\hbar}=R_{a b}^{\hbar}\left(z_{a}-z_{b}\right)
$$

(see (A.23) $)$ where $z_{a}, z_{b}, z_{c}, \hbar, \eta$ denote free generic complex parameters. Indices $a, b, c$ are distinct numbers of tensor components in $\operatorname{Mat}(N, \mathbb{C})^{\otimes n \geq 3} . R_{a b}^{\hbar}$ is defined in $a$-th and $b$-th components. If $c \neq a, b$ then $R_{a b}^{\hbar}$ acts on the $c$-th component of $\operatorname{Mat}(N, \mathbb{C})^{\otimes n}$ by $N \times N$ identity matrix.

The elliptic solution of (1.2) was found in [18]. It is the Baxter-Belavin's elliptic $\mathrm{GL}_{N}$ quantum $R$-matrix [4, 5] (in vector representation). The latter means that $R_{a b}^{\hbar}$ satisfies the quantum Yang-Baxter equation 1 :

$$
R_{a b}^{\hbar} R_{a c}^{\hbar} R_{b c}^{\hbar}=R_{b c}^{\hbar} R_{a c}^{\hbar} R_{a b}^{\hbar} .
$$

The property (1.2) of the Baxter-Belavin $R$-matrix was rediscovered in our papers [13, 14] in the framework of integrable systems and related topics - Painlevé equations, Schlesinger systems and their quantization via the Knizhnik-Zamolodchikov-Bernard (KZB) equations.

\footnotetext{
${ }^{1}$ See $[10]$ for reviews on the Yang-Baxter equation and related structures.
} 
Elliptic function identities. The associative Yang-Baxter equation (1.2) in the scalar case (when $N=1$ and $a, b, c=1,2,3$ ) is the Fay trisecant identity on elliptic curve

$$
\phi\left(z_{1}-z_{2}, \hbar\right) \phi\left(z_{2}-z_{3}, \eta\right)=\phi\left(z_{1}-z_{3}, \eta\right) \phi\left(z_{1}-z_{2}, \hbar-\eta\right)+\phi\left(z_{2}-z_{3}, \eta-\hbar\right) \phi\left(z_{1}-z_{3}, \hbar\right)
$$

The function $\phi(z, u)$ satisfying this equation is the Kronecker function. It is define as

$$
\phi(z, u)=\frac{\vartheta^{\prime}(0) \vartheta(z+u)}{\vartheta(z) \vartheta(u)}
$$

where $\vartheta(z)$ is the odd Riemann theta-function 2 (A.3). In this respect the Baxter-Belavin's $R$-matrix can be viewed as non-abelian generalization of the Kronecker function. In [14] we described a list of elliptic function identities and properties together with their $R$-matrix analogues. In particular, it is easy to verify that the Baxter-Belavin $R$-matrix defined as 3

$$
R_{12}^{\hbar}(z)=\sum_{\alpha \in \mathbb{Z}_{N} \times \mathbb{Z}_{N}} \varphi_{\alpha}\left(z, \omega_{\alpha}+\hbar\right) T_{\alpha} \otimes T_{-\alpha}, \quad \operatorname{Res}_{z=0} R_{12}^{\hbar}(z)=N P_{12},
$$

(see definition of $\varphi_{\alpha}\left(z, \omega_{\alpha}+\hbar\right)$ in (A.22) ) satisfies the skew-symmetry property

$$
R_{a b}^{\hbar}=-R_{b a}^{-\hbar}
$$

and the unitarity condition (see Appendix)

$$
R_{a b}^{\hbar} R_{b a}^{\hbar}=1 \otimes 1 N^{2}\left(\wp(N \hbar)-\wp\left(z_{a}-z_{b}\right)\right)
$$

where $\wp(z)$ is the Weierstrass $\wp$-function. Condition (1.7) is analogue of $\phi(z, u)=-\phi(-z,-u)$ while (1.8) is similar to (A.7) $\phi(z, u) \phi(z,-u)=\wp(z)-\wp(u)$.

In trigonometric (hyperbolic) or rational cases the Weierstrass $\wp$-function entering (1.8) equals $\wp(z)=1 / \sinh ^{2}(z)$ or $\wp(z)=1 / z^{2}$ respectively. The simplest example of the $R$-matrix satisfying (1.2), (1.3) and (1.7), (1.8) is the quantum Yang's $R$-matrix [23]:

$$
R_{12}^{\mathrm{Yang}}(z)=\frac{1 \otimes 1}{\hbar}+\frac{N P_{12}}{z}
$$

It is the $R$-matrix analogue of function $1 / \hbar+1 / z$. The class of $R$-matrices under consideration includes nontrivial trigonometric and rational degenerations (of (1.6)), which satisfy (1.2), (1.7) and (1.8). These type $R$-matrices can be found in [2] and [22, 15] for trigonometric and rational cases respectively. In $\mathrm{GL}_{2}$ case the corresponding 7 -vertex and 11-vertex $R$-matrices were found in [7]. See also [6] which results are (presumably) gauge equivalent to those of [2, 22, 15].

Purpose of the paper.

1. Yang-Baxter equation with two Planck constants. As we will see it follows from (1.2), (1.7) and (1.8) that the quantum $R$-matrix satisfies also cubic equations of Yang-Baxter type. The one closest to the original one (1.3) have the following form:

$$
R_{12}^{\eta} R_{13}^{\hbar} R_{23}^{\eta}+R_{12}^{\hbar} R_{13}^{\eta} R_{23}^{\hbar}=R_{23}^{\eta} R_{13}^{\hbar} R_{12}^{\eta}+R_{23}^{\hbar} R_{13}^{\eta} R_{12}^{\hbar}
$$

\footnotetext{
${ }^{2}$ In trigonometric (hyperbolic) or rational cases $\phi(z, u)=\operatorname{coth}(z)+\operatorname{coth}(u)$ or $\phi(z, u)=1 / z+1 / u$ respectively.

${ }^{3}$ In [18] this $R$-matrix was found in different - Richey-Tracy form [19].
} 
where again $R_{a b}^{\hbar}=R_{a b}^{\hbar}\left(z_{a}-z_{b}\right)$. When $\hbar=\eta(1.10)$ coincides with the Yang-Baxter equation (1.3). Another (more general) type of equation contains $\wp$-function entering the unitarity condition (1.8):

$$
R_{12}^{\eta} R_{13}^{\hbar} R_{23}^{\eta}-R_{23}^{\hbar} R_{13}^{\eta} R_{12}^{\hbar}=R_{13}^{\hbar+\eta} N^{2}(\wp(N \eta)-\wp(N \hbar)) .
$$

Let us mention that equations of (1.11) type were considered in [20] in the context of double Lie (and the quadratic Poisson) structures.

2. Quantum algebras. Given a quantum $R$-matrix one can define the following (Sklyanin type) algebra using exchange relations:

$$
R_{12}^{\hbar}(z-w) \hat{L}_{1}^{\hbar}(z, \hat{S}) \hat{L}_{2}^{\hbar}(w, \hat{S})=\hat{L}_{2}^{\hbar}(w, \hat{S}) \hat{L}_{1}^{\hbar}(z, \hat{S}) R_{12}^{\hbar}(z-w)
$$

with $\hat{L}^{\hbar}(z, \hat{S})=\operatorname{tr}_{2}\left(R_{12}^{\hbar}(z) \hat{S}_{2}\right)$. For $N=2$, i.e. in the Baxter's case equation (1.12) provides the Sklyanin algebra [21] for the components $\hat{S}_{\alpha}, \hat{S}=\sum_{\alpha} \hat{S}_{\alpha} T_{\alpha}$. In particular case (see (3.3)) such $L$-operator is just the $R$-matrix (1.6) itself, and the exchange relations (1.12) are fulfilled identically due to Yang-Baxter equation (1.3). It means that (1.12) follows from (1.3) by treating one of tensor components as some Sklyanin algebra module.

In the same way we can define exchange relations corresponding to (1.10) as follows:

$$
\begin{aligned}
R_{12}^{\hbar}(z-w) \hat{L}_{1}^{\eta}\left(z, \hat{S}^{\eta}\right) & \hat{L}_{2}^{\hbar}\left(w, \hat{S}^{\hbar}\right)+R_{12}^{\eta}(z-w) \hat{L}_{1}^{\hbar}\left(z, \hat{S}^{\hbar}\right) \hat{L}_{2}^{\eta}\left(w, \hat{S}^{\eta}\right)= \\
& =\hat{L}_{2}^{\hbar}\left(w, \hat{S}^{\hbar}\right) \hat{L}_{1}^{\eta}\left(z, \hat{S}^{\eta}\right) R_{12}^{\hbar}(z-w)+\hat{L}_{2}^{\eta}\left(w, \hat{S}^{\eta}\right) \hat{L}_{1}^{\hbar}\left(z, \hat{S}^{\hbar}\right) R_{12}^{\eta}(z-w)
\end{aligned}
$$

Again, when $\hbar=\eta$ it coincides with the ordinary exchange relations (1.12). As we will see this equation defines coupled Sklyanin algebras, i.e. given a pair of Sklyanin algebras for $\hat{S}^{\hbar}$ and $\hat{S}^{\eta}$ the equation (1.13) provides commutation relations between $\hat{S}^{\hbar}$ and $\hat{S}^{\eta}$.

3. Symmetric $R$-matrix in $\operatorname{Mat}(N, \mathbb{C})^{\otimes 2} \otimes \operatorname{Mat}(M, \mathbb{C})^{\otimes 2}$. Here we consider the following extension of the Baxter-Belavin $R$-matrix (1.6):

$$
\Re_{12, \tilde{1} \tilde{2}}(z, \hbar)=\sum_{\tilde{\alpha} \in \mathbb{Z}_{M} \times \mathbb{Z}_{M}} \exp \left(2 \pi \imath N \frac{\tilde{\alpha}_{2}}{M} \hbar\right) R_{12}^{\hbar}\left(z+N \omega_{\tilde{\alpha}}\right) \otimes \tilde{T}_{\tilde{\alpha}} \otimes \tilde{T}_{-\tilde{\alpha}}
$$

where $\tilde{T}_{\tilde{\alpha}}$ is $T_{\alpha}$ of size $M \times M$. We call this $R$-matrix symmetric in the sense that both arguments (the Planck constant $\hbar$ and the spectral parameter $z$ ) are averaged over lattices $\mathbb{Z}_{N} \times \mathbb{Z}_{N}$ and $\mathbb{Z}_{M} \times \mathbb{Z}_{M}$ respectively. In the simplest rational case corresponding to Yang's $R$-matrix (1.9) the expression (1.14) is reduced to

$$
\mathfrak{R}_{12, \tilde{1} \tilde{2}}(z, \hbar)=M \frac{1_{N} \otimes 1_{N} \otimes \tilde{P}_{\tilde{1} \tilde{2}}}{\hbar}+N \frac{P_{12} \otimes \tilde{1}_{M} \otimes \tilde{1}_{M}}{z} .
$$

The limiting cases $N=1$ or $M=1$ are given as follows:

$$
\begin{aligned}
& M_{M=1} \swarrow \mathfrak{\Re}_{12, \tilde{1} \tilde{2}}(z, \hbar) \searrow^{N=1} \\
& R_{12}^{\hbar}(z) \quad R_{\tilde{1} \tilde{2}}^{z}(\hbar)
\end{aligned}
$$


We will show that such an $R$-matrix satisfies a set of relations similar to those for the ordinary $R$-matrix. In particular, we have the following generalization of the associative Yang-Baxter equation (1.4):

$$
\mathfrak{R}_{12, \tilde{1} \tilde{2}} \mathfrak{R}_{23, \tilde{3} \tilde{2}}=\mathfrak{R}_{13, \tilde{3} \tilde{2}} \mathfrak{R}_{12, \tilde{1} \tilde{3}}+\mathfrak{R}_{23, \tilde{3} \tilde{1}} \mathfrak{R}_{13, \tilde{1} \tilde{2}}
$$

where $\mathfrak{R}_{a b, \tilde{a} \tilde{b}}=\mathfrak{R}_{a b, \tilde{a} \tilde{b}}\left(z_{a}-z_{b}, \hbar_{\tilde{a}}-\hbar_{\tilde{b}}\right)$. The cubic equations arise similarly. For example, the generalizations of (1.11) takes the form:

$$
\begin{aligned}
& \mathfrak{R}_{12, \tilde{3} \tilde{2}} \mathfrak{R}_{13, \tilde{1} \tilde{3}} \mathfrak{R}_{23, \tilde{3} \tilde{2}}=\mathfrak{R}_{23, \tilde{1} \tilde{3}} \mathfrak{R}_{13, \tilde{3} \tilde{2}} \mathfrak{R}_{12, \tilde{1} \tilde{3}}+N^{2} M^{2} \mathfrak{R}_{13, \tilde{1} \tilde{2}}\left(\wp\left(N \hbar_{\tilde{3} \tilde{2}}\right)-\wp\left(N \hbar_{\tilde{1} \tilde{3}}\right)\right), \\
& \mathfrak{R}_{32, \tilde{1} \tilde{2}} \mathfrak{R}_{13, \tilde{1} \tilde{3}} \mathfrak{R}_{32, \tilde{2} \tilde{3}}=\mathfrak{R}_{13, \tilde{2} \tilde{3}} \mathfrak{R}_{32, \tilde{1} \tilde{3}} \mathfrak{R}_{13, \tilde{1} \tilde{2}}+N^{2} M^{2} \mathfrak{R}_{12, \tilde{1} \tilde{3}}\left(\wp\left(M z_{23}\right)-\wp\left(M z_{13}\right)\right) .
\end{aligned}
$$

where $z_{a b}=z_{a}-z_{b}$ and $\hbar_{\tilde{a} \tilde{b}}=\hbar_{\tilde{a}}-\hbar_{\tilde{b}}$. The Yang-Baxter like equation (1.10) is generalized as follows:

$$
\begin{aligned}
& \mathfrak{R}_{12, \tilde{3} \tilde{2}}\left(z_{12}, \hbar_{\tilde{3} \tilde{2}}\right) \mathfrak{R}_{13, \tilde{1} \tilde{3}}\left(z_{13}, \hbar_{\tilde{1} \tilde{3}}\right) \mathfrak{R}_{23, \tilde{3} \tilde{2}}\left(z_{23}, \hbar_{\tilde{3} \tilde{2}}\right)+\mathfrak{R}_{12, \tilde{3} \tilde{2}}\left(z_{12}, \hbar_{\tilde{1} \tilde{3}}\right) \mathfrak{R}_{13, \tilde{1} \tilde{3}}\left(z_{13}, \hbar_{\tilde{3} \tilde{2}}\right) \mathfrak{R}_{23, \tilde{3} \tilde{2}}\left(z_{23}, \hbar_{\tilde{1} \tilde{3}}\right) \\
& =\mathfrak{R}_{23, \tilde{1} \tilde{3}}\left(z_{23}, \hbar_{\tilde{1} \tilde{3}}\right) \mathfrak{R}_{13, \tilde{3} \tilde{2}}\left(z_{13}, \hbar_{\tilde{3} \tilde{2}}\right) \mathfrak{R}_{12, \tilde{1} \tilde{3}}\left(z_{12}, \hbar_{\tilde{1} \tilde{3}}\right)+\mathfrak{R}_{23, \tilde{1} \tilde{3}}\left(z_{23}, \hbar_{\tilde{3} \tilde{2}}\right) \mathfrak{R}_{13, \tilde{3} \tilde{2}}\left(z_{13}, \hbar_{\tilde{1} \tilde{3}}\right) \mathfrak{R}_{12, \tilde{1} \tilde{3}}\left(z_{12}, \hbar_{\tilde{3} \tilde{2}}\right)
\end{aligned}
$$

and

$$
\begin{aligned}
& \Re_{32, \tilde{1} \tilde{2}}\left(z_{32}, \hbar_{\tilde{1} \tilde{2}}\right) \mathfrak{R}_{13, \tilde{1} \tilde{3}}\left(z_{13}, \hbar_{\tilde{1} \tilde{3}}\right) \mathfrak{R}_{32, \tilde{2} \tilde{3}}\left(z_{32}, \hbar_{\tilde{2} \tilde{3}}\right)+\mathfrak{R}_{32, \tilde{1} \tilde{2}}\left(z_{13}, \hbar_{\tilde{1} \tilde{2}}\right) \mathfrak{R}_{32, \tilde{1} \tilde{3}}\left(z_{13}, \hbar_{\tilde{1} \tilde{3}}\right) \mathfrak{R}_{32, \tilde{2} \tilde{3}}\left(z_{13}, \hbar_{\tilde{2} \tilde{3}}\right) \\
& =\mathfrak{R}_{13, \tilde{2} \tilde{3}}\left(z_{13}, \hbar_{\tilde{2} \tilde{3}}\right) \mathfrak{R}_{32, \tilde{1} \tilde{3}}\left(z_{32}, \hbar_{\tilde{1} \tilde{3}}\right) \mathfrak{R}_{13, \tilde{1} \tilde{2}}\left(z_{13}, \hbar_{\tilde{1} \tilde{2}}\right)+\mathfrak{R}_{13, \tilde{2} \tilde{3}}\left(z_{32}, \hbar_{\tilde{2} \tilde{3}}\right) \mathfrak{R}_{32, \tilde{1} \tilde{3}}\left(z_{13}, \hbar_{\tilde{1} \tilde{3} \tilde{3}}\right) \mathfrak{R}_{13, \tilde{1} \tilde{2}}\left(z_{32}, \hbar_{\tilde{1} \tilde{2}}\right)
\end{aligned}
$$

Acknowledgments. The work was supported by RFBR grant 15-01-04217 and by joint RFBR project 15-51-52031 $\mathrm{HHC}_{a}$. The work of A.L. was partially supported by Department of Mathematics NRU HSE, the subsidy granted to the HSE by the Government of the Russian Federation for the implementation of the Global Competitiveness Program and by the Simons Foundation. The work of A.Z. was also partially supported by the D. Zimin's fund "Dynasty".

\section{Yang-Baxter equations with two Planck constants}

Consider a quantum $R$-matrix which satisfies the associative Yang-Baxter equation (1.2) and the properties (1.7), (1.8). Then it is easy to get a set of cubic (in $R$ ) relations.

Proposition 2.1 The following cubic relations are valid for a common solution of (1.2), (1.7), (1.8):

$$
\begin{gathered}
R_{12}^{\hbar} R_{13}^{\hbar} R_{23}^{\hbar}=R_{23}^{\hbar} R_{13}^{\hbar} R_{12}^{\hbar} \\
R_{a b}^{\hbar} R_{b c}^{\hbar} R_{c a}^{\hbar}+R_{a c}^{\hbar} R_{c b}^{\hbar} R_{b a}^{\hbar}=-N^{3} \wp^{\prime}(N \hbar) 1_{a} \otimes 1_{b} \otimes 1_{c}, \\
R_{a b}^{\eta} R_{a c}^{\hbar} R_{b c}^{\eta}-R_{b c}^{\hbar} R_{a c}^{\eta} R_{a b}^{\hbar}=R_{a c}^{\hbar+\eta} N^{2}(\wp(N \eta)-\wp(N \hbar)), \\
R_{a b}^{\eta} R_{a c}^{\hbar} R_{b c}^{\eta}+R_{a b}^{\hbar} R_{a c}^{\eta} R_{b c}^{\hbar}=R_{b c}^{\eta} R_{a c}^{\hbar} R_{a b}^{\eta}+R_{b c}^{\hbar} R_{a c}^{\eta} R_{a b}^{\hbar},
\end{gathered}
$$

where $R_{a b}^{\hbar}=R_{a b}^{\hbar}\left(z_{a}-z_{b}\right)$ and $a, b, c$ are distinct numbers from the set $\{1,2,3\}$. 
Proof: First, notice that the primary one is the third identity (2.3) since all others follow from it. Indeed, the Yang-Baxter equation (2.1) follows from (2.3) in the case $\hbar=\eta$.

The second equation (2.2) appears from (2.3) in the limit $\eta \rightarrow-\hbar$ : one should use the classical limit (A.25), and then the skew-symmetry property (1.7) to arrange the indices in a cyclic order. This gives (2.2) up to permutation of indices.

The fourth relation (2.4) can be called Yang-Baxter equation with two Planck constants because its structure is similar to (2.1) and it is coincide with (2.1) for $\hbar=\eta$. It is easy to see that (2.4) follows from (2.3) as skew symmetry of (2.3) l.h.s. with respect to interchanging $\hbar$ and $\eta$.

Thus we need to prove (2.3) . Consider (1.2) and multiply both parts by $R_{b c}^{\hbar-\eta}$ from the left side:

$$
R_{b c}^{\hbar-\eta} R_{a b}^{\hbar} R_{b c}^{\eta}=R_{b c}^{\hbar-\eta} R_{a c}^{\eta} R_{a b}^{\hbar-\eta}+R_{b c}^{\hbar-\eta} R_{b c}^{\eta-\hbar} R_{a c}^{\hbar} .
$$

Now interchange the indices 2,3 in (1.2) and multiply both parts by $R_{b c}^{\eta}$

$$
R_{a c}^{\hbar} R_{c b}^{\eta} R_{b c}^{\eta}=R_{a b}^{\eta} R_{a c}^{\hbar-\eta} R_{b c}^{\eta}-R_{b c}^{\hbar-\eta} R_{a b}^{\hbar} R_{b c}^{\eta}
$$

where in the last term we have already used $R_{c b}^{\eta-\hbar}=-R_{b c}^{\hbar-\eta}$. From (1.7), (1.8) it follows that $R_{b c}^{\hbar-\eta} R_{b c}^{\eta-\hbar}$ and $R_{c b}^{\eta} R_{b c}^{\eta}$ are scalar operators. Subtracting (2.6) from (2.5) we get (2.3) with $\hbar:=\hbar-\eta$.

Let us also remark that the identity (2.2) appeared to be related to $R$-matrix valued Lax operator for the classical Calogero-Moser model [13]. Consider the following block-matrix:

$$
\mathcal{L}(\hbar)=\sum_{a, b=1}^{n} \tilde{E}_{a b} \otimes\left(1-\delta_{a b}\right) R_{a b}^{\hbar}\left(z_{a}-z_{b}\right),
$$

where $\tilde{E}_{a b}$ is the standard basis in $\operatorname{Mat}(n, \mathbb{C})$, i.e. $\left(\tilde{E}_{a b}\right)_{c d}=\delta_{a c} \delta_{b d}$. Then the diagonal blocks of $\operatorname{tr} \mathcal{L}^{k}(\hbar)$ are scalar operators, and the scalar functions are obtained as if $\mathcal{L}(\hbar)$ were element of $\operatorname{Mat}(n, \mathbb{C})$ with matrix elements $l_{a b}(\hbar)=\left(1-\delta_{a b}\right) N \phi\left(N \hbar, z_{a}-z_{b}\right)$ :

$$
\left(\mathcal{L}^{k}(\hbar)\right)_{a a}=1 \otimes \ldots \otimes 1\left(l^{k}(\hbar)\right)_{a a} .
$$

When $n=2$ this equation is equivalent to the unitarity condition (1.8), while for $n=3$ (2.8) reproduces (2.2).

One more application of (2.2) comes from the classical limit (A.25). The identities which appear from (2.2) together with (A.29) provide sufficient conditions for compatibility of the KZB connections (see details in [13]).

Finally we conclude that the identity (2.3) is of great importance. On one hand it reproduces the Yang-Baxter equations, and in this sense it "knows" about quantum integrability and related algebraic structures including quantum groups, Sklyanin algebras, e.t.c. On the other hand (2.3) also "knows" about classical integrable system of Calogero type. At last, the same identity provides the quantization of the Schlesinger systems via compatibility of the KZB connections. 


\section{Quantum algebras}

In this section we discuss differen types of quadratic finite-dimensional quantum algebras arising from exchange like relations. The quantum (Lax) L-operator [21, 12] is defined as

$$
\hat{L}^{\hbar}(z, \hat{S})=\hat{L}^{\hbar}(z)=\operatorname{tr}_{2}\left(R_{12}^{\hbar}(z) \hat{S}_{2}\right), \quad \hat{S}=\sum_{\alpha} T_{\alpha} \hat{S}_{\alpha}
$$

where $\left\{\hat{S}_{\alpha}, \alpha \in \mathbb{Z}_{N}^{\times 2}\right\}$ is the set of generators of the quantum algebra $\mathcal{A}$. For the elliptic $R$-matrix (1.6) we have

$$
\hat{L}^{\hbar}(z)=\sum_{\alpha} T_{\alpha} \hat{S}_{\alpha} \varphi_{\alpha}\left(z, \omega_{\alpha}+\hbar\right)
$$

For the vector representation $\rho_{N}$ of $\mathcal{A}$ given by

$$
\rho_{N}\left(\hat{S}_{\alpha}\right)=T_{-\alpha} \in \operatorname{Mat}(N, \mathbb{C})
$$

the Lax operator (3.2) coincides with the $R$-matrix (1.6).

Finite Heisenberg group (see (A.18) in Appendix) is the simplest example of quantum algebra which comes from $R$-matrix relations for the $L$-operator (3.1).

Proposition 3.1 Relations

$$
\hat{L}_{1}^{\hbar}(z) \hat{L}_{2}^{\eta}(w)=\hat{L}_{2}^{\hbar+\eta}(w) R_{12}^{\hbar}(z-w)-R_{12}^{-\eta}(z-w) \hat{L}_{1}^{\hbar+\eta}(z)
$$

or

$$
\hat{L}_{2}^{\eta}(w) \hat{L}_{1}^{\hbar}(z)=R_{12}^{\hbar}(z-w) \hat{L}_{2}^{\hbar+\eta}(w)-\hat{L}_{1}^{\hbar+\eta}(z) R_{12}^{-\eta}(z-w)
$$

with L-operator defined in (3.2) and $R$-matrix (1.6) are equivalent to

$$
\hat{S}_{1} \hat{S}_{2}=N P_{12} \hat{S}_{1}
$$

i.e.

$$
\hat{S}_{\alpha} \hat{S}_{\beta}=\kappa_{\alpha, \beta} \hat{S}_{\alpha+\beta}
$$

in components. The associativity condition for the triple product $\hat{L}_{1}^{\hbar}(z) \hat{L}_{2}^{\eta}(w) \hat{L}_{3}^{\xi}(x)$ follows from the associative Yang-Baxter equation (1.2).

The proof of (3.4), (3.5) follows directly from the Fay identity (1.4). The statement about associativity is analogues to the derivation of (1.1) in [1].

Notice that the commutation relations

$$
\left[\hat{L}_{1}^{\hbar}(z), \hat{L}_{2}^{\eta}(w)\right]=\left[\hat{L}_{1}^{\hbar+\eta}(z), R_{12}^{-\eta}(z-w)\right]+\left[\hat{L}_{2}^{\hbar+\eta}(w), R_{12}^{\hbar}(z-w)\right]
$$

are equivalent to those for the Lie algebra $\mathrm{gl}_{N}$ :

$$
\left[\hat{S}_{1}, \hat{S}_{2}\right]=N\left[P_{12}, \hat{S}_{1}\right]
$$

Obviously the same expression (r.h.s. of (3.8)) can be used for definition of the Poisson-Lie brackets $\left\{S_{1}, S_{2}\right\}=N\left[P_{12}, S_{1}\right]$ on $\mathrm{gl}_{N}^{*}$ :

$$
\left\{L_{1}^{\hbar}(z), L_{2}^{\eta}(w)\right\}=\left[L_{1}^{\hbar+\eta}(z), R_{12}^{-\eta}(z-w)\right]+\left[L_{2}^{\hbar+\eta}(w), R_{12}^{\hbar}(z-w)\right] .
$$


with the classical matrix-valued function $L(z, S)$ on the phase space $\mathrm{gl}_{N}^{*}$ parameterized by $S_{\alpha}$ $\left(S=\sum_{\alpha} S_{\alpha} T_{\alpha}\right)$. The Jacobi identity is due to (1.2). The relation (3.10) differs from the custom classical exchange relations

$$
\left\{l_{1}(z), l_{2}(w)\right\}=\left[l_{1}(z), r_{12}(z-w)\right]+\left[l_{2}(w), r_{12}(z-w)\right]
$$

where the classical $r$-matrix $r_{12}(z)$ (A.25) is used, and the Jacobi identity is fulfilled due the classical Yang-Baxter equation (A.26) for $r_{12}(z)$. Alternatively, we can say that in the case under consideration the constant $\hbar$ (and $\eta$ ) is not the Planck constant entering the quantum $R$-matrix but rather additional spectral parameter entering the classical $r$-matrix. Indeed, the final commutation relations (3.9) are independent of $\hbar, \eta$ (as well as they are independent of $z$ and $w$ ). Such interpretation is close to the consideration suggested in [18].

Exchange relations with two Planck constants and coupled Sklyanin algebras. Consider a pair of the quantum Lax operators

$$
\hat{L}^{\hbar}(z)=\hat{L}^{\hbar}\left(z, \hat{S}^{\hbar}\right)=\sum_{\alpha} T_{\alpha} \hat{S}_{\alpha}^{\hbar} \varphi_{\alpha}\left(z, \omega_{\alpha}+\hbar\right)
$$

and

$$
\hat{L}^{\eta}(z)=\hat{L}^{\eta}\left(z, \hat{S}^{\eta}\right)=\sum_{\alpha} T_{\alpha} \hat{S}_{\alpha}^{\eta} \varphi_{\alpha}\left(z, \omega_{\alpha}+\eta\right)
$$

Each of them defines its own Sklyanin algebra via exchange relations (1.12). See details in the Appendix. Here we suggest another exchange type relation which provides commutation relations between $\hat{S}^{\hbar}$ and $\hat{S}^{\eta}$. It is of the form:

$$
\begin{aligned}
& R_{12}^{\hbar}(z-w) \hat{L}_{1}^{\eta}(z) \hat{L}_{2}^{\hbar}(w)+R_{12}^{\eta}(z-w) \hat{L}_{1}^{\hbar}(z) \hat{L}_{2}^{\eta}(w)= \\
& =\hat{L}_{2}^{\hbar}(w) \hat{L}_{1}^{\eta}(z) R_{12}^{\hbar}(z-w)+\hat{L}_{2}^{\eta}(w) \hat{L}_{1}^{\hbar}(z) R_{12}^{\eta}(z-w)
\end{aligned}
$$

Proposition 3.2 Equation (3.14) for the Lax operators $\hat{L}^{\hbar}\left(\hat{S}^{\hbar}\right)$ and $\hat{L}^{\eta}\left(\hat{S}^{\eta}\right)$ is equivalent to the following commutation relations for the components of $\hat{S}^{\hbar}$ and $\hat{S}^{\eta}$ :

$$
\sum_{\gamma} \kappa_{\gamma, \alpha-\beta} \hat{S}_{\alpha-\gamma}^{\eta} \hat{S}_{\beta+\gamma}^{\hbar} \mathrm{f}_{\alpha, \beta, \gamma}^{\hbar, \eta}+\kappa_{\gamma, \alpha-\beta} \hat{S}_{\alpha-\gamma}^{\hbar} \hat{S}_{\beta+\gamma}^{\eta} \mathrm{f}_{\alpha, \beta, \gamma}^{\eta, \hbar}=0
$$

where the structure constants $\mathrm{f}_{\alpha, \beta, \gamma}^{\hbar, \eta}$ are given by

$$
\begin{array}{ll}
\text { for } \beta \neq 0: & \mathrm{f}_{\alpha, \beta, \gamma}^{\hbar, \eta}=E_{1}\left(\omega_{\gamma}+\hbar\right)-E_{1}\left(\omega_{\alpha-\beta-\gamma}+\eta\right)+E_{1}\left(\omega_{\alpha-\gamma}+\eta\right)-E_{1}\left(\omega_{\beta+\gamma}+\hbar\right), \\
\text { for } \beta=0: & \mathrm{f}_{\alpha, 0, \gamma}^{\hbar, \eta}=\wp\left(\omega_{\gamma}+\hbar\right)-\wp\left(\omega_{\alpha-\gamma}+\eta\right) .
\end{array}
$$

$\underline{\text { Proof: }}$ Here we use short notation $\varphi_{\alpha}^{\hbar}(z)=\varphi_{\alpha}^{\hbar}\left(z, \omega_{\alpha}+\hbar\right)$. Compute

$$
\begin{gathered}
R_{12}^{\hbar}(z-w) \hat{L}_{1}^{\eta}(z) \hat{L}_{2}^{\hbar}(w)-\hat{L}_{2}^{\eta}(w) \hat{L}_{1}^{\hbar}(z) R_{12}^{\eta}(z-w)=\sum_{\alpha, \beta, \gamma} T_{\alpha} \otimes T_{\beta} \times \\
\times\left(\hat{S}_{\alpha-\gamma}^{\eta} \hat{S}_{\beta+\gamma}^{\hbar} \kappa_{\gamma, \alpha-\beta} \varphi_{\alpha-\gamma}^{\eta}(z) \varphi_{\beta+\gamma}^{\hbar}(w) \varphi_{\gamma}^{\hbar}(z-w)-\hat{S}_{\beta+\gamma}^{\eta} \hat{S}_{\alpha-\gamma}^{\hbar} \kappa_{\alpha-\beta, \gamma} \varphi_{\alpha-\gamma}^{\hbar}(z) \varphi_{\beta+\gamma}^{\eta}(w) \varphi_{\gamma}^{\eta}(z-w)\right)=
\end{gathered}
$$


By redefining the summation index for the second term as $\gamma \rightarrow \alpha-\beta-\gamma$ we get for the following coefficient behind the tensor component $T_{\alpha} \otimes T_{\beta}$ :

$$
\begin{gathered}
=\sum_{\alpha, \beta, \gamma} T_{\alpha} \otimes T_{\beta} \hat{S}_{\alpha-\gamma}^{\eta} \hat{S}_{\beta+\gamma}^{\hbar} \kappa_{\gamma, \alpha-\beta}\left(\varphi_{\alpha-\gamma}^{\eta}(z) \varphi_{\beta+\gamma}^{\hbar}(w) \varphi_{\gamma}^{\hbar}(z-w)-\varphi_{\beta+\gamma}^{\hbar}(z) \varphi_{\alpha-\gamma}^{\eta}(w) \varphi_{\alpha-\beta-\gamma}^{\eta}(z-w)\right) \\
=\sum_{\alpha, \beta, \gamma} T_{\alpha} \otimes T_{\beta} \hat{S}_{\alpha-\gamma}^{\eta} \hat{S}_{\beta+\gamma}^{\hbar} \kappa_{\gamma, \alpha-\beta} \mathrm{f}_{\alpha, \beta, \gamma}^{\hbar, \eta} \varphi_{\alpha}^{\hbar+\eta}(z) \varphi_{\beta}(w),
\end{gathered}
$$

where we assume $\varphi_{0}(w)=1$. The last equality follows from (A.8), (A.9). In this way we reproduce the first term in (3.15). The second term in (3.15) is obtained in the same way by interchanging $\hbar$ and $\eta$.

Notice that when $\hbar=\eta$ the structure constants $\mathrm{f}_{\alpha, \beta, \gamma}^{\hbar, \hbar}=\mathrm{f}_{\alpha, \beta, \gamma}^{\hbar}$ coincide with those for the Sklyanin algebra (A.31).

Recently the coupled Sklyanin algebras appeared also in different way - via the modular double [9]. In that case two algebras depend on different modular parameters $\tau$. In our case this parameter is the same but the Planck constants are different.

Remark. One can also consider (quasi)classical limits of the Yang-Baxter (2.4) and exchange relations (3.14). There are different possibilities for the limits since we deal with two Planck constants. In particular, when $\hbar \rightarrow 0$ (while $\eta$ is finite) (2.4) is reduced to

$$
\left[R_{12}^{\eta}, R_{23}^{\eta}\right]+\left[R_{13}^{\eta}, r_{23}\right]+\left[r_{12}, R_{13}^{\eta}\right]=0
$$

where $r_{a b}$ is the classical $r$-matrix (A.25). At this stage the generators $\hat{S}_{\alpha}^{\hbar}$ become classical variables $S_{\alpha}$ while $\hat{S}_{\alpha}^{\eta}$ are still quantum. Then we get a "half-quantum" Poisson structure between the commutative classical variables $S_{\alpha}$ and noncommutative variables $\hat{S}_{\beta}^{\eta}$. Taking then limit $\eta \rightarrow 0$ the equation (3.17) is reduced to the ordinary classical Yang-Baxter equation (A.26), and the Poisson structure is the standard classical Sklyanin algebra. We will describe the quasiclassical limits in our next paper.

\section{$4 \quad$ Symmetric $R$-matrix in $\operatorname{Mat}(N, \mathbb{C})^{\otimes 2} \otimes \operatorname{Mat}(M, \mathbb{C})^{\otimes 2}$}

In this section we consider an extension of the $R$-matrix (1.6) in $\operatorname{Mat}(N, \mathbb{C})^{\otimes 2}$ to the matrix function in $\operatorname{Mat}(N, \mathbb{C})^{\otimes 2} \otimes \operatorname{Mat}(M, \mathbb{C})^{\otimes 2}$. This type of $R$-matrices (see e.g. [11, 16, 3]) appears naturally when $N:=N \times M$ from the original $\operatorname{Mat}(N, \mathbb{C})^{\otimes 2}$-valued one. Our definition is slightly modified in a way to get expression which is symmetric in spectral parameter $z$ and the Planck constant $\hbar$. The simplest example of the $R$-matrix which we are going to discuss here can be given in the rational case. Consider the following expression:

$$
\begin{gathered}
\Re_{12, \tilde{1} \tilde{2}}\left(z_{1}-z_{2}, \hbar_{\tilde{1}}-\hbar_{\tilde{2}}\right)=M \frac{1_{N} \otimes 1_{N} \otimes \tilde{P}_{\tilde{1} \tilde{2}}}{\hbar_{\tilde{1}}-\hbar_{\tilde{2}}}+N \frac{P_{12} \otimes \tilde{1}_{M} \otimes \tilde{1}_{M}}{z_{1}-z_{2}}, \\
\mathfrak{R}_{12, \tilde{1} \tilde{2}}\left(z_{1}-z_{2}, \hbar_{\tilde{1}}-\hbar_{\tilde{2}}\right) \in \operatorname{Mat}(N, \mathbb{C})^{\otimes 2} \otimes \operatorname{Mat}(M, \mathbb{C})^{\otimes 2}
\end{gathered}
$$

where $\tilde{P}_{\tilde{1} \tilde{2}}$ is the permutation operator in $\operatorname{Mat}(M, \mathbb{C})^{\otimes 2}$, and $1_{N}\left(\right.$ or $\left.\tilde{1}_{M}\right)$ is the identity matrix in $\operatorname{Mat}(N, \mathbb{C})($ or in $\operatorname{Mat}(M, \mathbb{C})) . \mathfrak{R}_{12, \tilde{1} \tilde{2}}$ has four tensor indices. The first pair is for the components 
of $\operatorname{Mat}(N, \mathbb{C})^{\otimes 2}$, and the second pair is for the components of $\operatorname{Mat}(M, \mathbb{C})^{\otimes 2}$. When $M=1$ (4.1) coincides with the Yang's $R$-matrix (1.9) $R_{12}^{\hbar_{1}-\hbar_{\tilde{2}}}\left(z_{1}-z_{2}\right) \in \operatorname{Mat}(N, \mathbb{C})^{\otimes 2}$. In the same way when $N=1$ (4.1) gives $R_{12}^{z_{1}-z_{2}}\left(\hbar_{\tilde{1}}-\hbar_{\tilde{2}}\right) \in \operatorname{Mat}(M, \mathbb{C})^{\otimes 2}$.

The expression (4.1) is not a new $R$-matrix of course. Multiplying it by $1_{N} \otimes 1_{N} \otimes \tilde{P}_{\tilde{1} \tilde{2}}$ we get a special form of the Yang's $R$-matrix in $\operatorname{Mat}(N M, \mathbb{C})^{\otimes 2}$.

Below we define elliptic analogue of (4.1). But first we recall some important properties of the Baxter-Belavin's $R$-matrix which will be to extended to the case $\operatorname{Mat}(N, \mathbb{C})^{\otimes 2} \otimes \operatorname{Mat}(M, \mathbb{C})^{\otimes 2}$ for the proper definition of underlying set of functions. To be exact, our goal is to define a set of functions $\Phi_{\alpha, \tilde{\alpha}}(z, \hbar), \alpha \in \mathbb{Z}_{N} \times \mathbb{Z}_{N}, \tilde{\alpha} \in \mathbb{Z}_{M} \times \mathbb{Z}_{M}$ which are invariant with respect to shift by the full lattice periods $\alpha \rightarrow \alpha+N, \alpha \rightarrow \alpha+N \tau$ and $\tilde{\alpha} \rightarrow \tilde{\alpha}+M, \tilde{\alpha} \rightarrow \tilde{\alpha}+M \tau$.

For the reasons given above (and for the brevity sake) we will refer to $\mathfrak{R}_{12, \tilde{1} \tilde{2}}$ type expression as symmetric $R$-matrix though it is not such symmetric in the elliptic case as in (4.1).

Symmetries of Baxter-Belavin $R$-matrix. The quantum elliptic $R$-matrix (1.6) is $\mathbb{Z}_{N} \times \mathbb{Z}_{N}$ symmetric, i.e.

$$
R_{12}^{\hbar}(z)=g_{1}^{-1} g_{2}^{-1} R_{12}^{\hbar}(z) g_{1} g_{2}, \quad g_{1}=g \otimes 1, g_{2}=1 \otimes g,
$$

where $g=Q$ or $g=\Lambda$ (A.14). This $R$-matrix is the quasiperiodic function of the spectral parameter $z$

$$
\begin{gathered}
R_{12}^{\hbar}(z+1)=Q_{1}^{-1} R_{12}^{\hbar}(z) Q_{1}, \\
R_{12}^{\hbar}(z+\tau)=\exp (-2 \pi \imath \hbar) \Lambda_{1}^{-1} R_{12}^{\hbar}(z) \Lambda_{1}
\end{gathered}
$$

on the elliptic curve with periods $1, \tau$. At the same time it is the quasiperiodic function of the Planck constant $\hbar$ on the smaller elliptic curve with periods $1 / N, \tau / N$ :

$$
\begin{gathered}
R_{12}^{\hbar+1 / N}(z)=Q_{1}^{-1} R_{12}^{\hbar}(z) Q_{2}, \\
R_{12}^{\hbar+\tau / N}(z)=\exp (-2 \pi \imath z / N) \Lambda_{1}^{-1} R_{12}^{\hbar}(z) \Lambda_{2} .
\end{gathered}
$$

The property (4.4) can be considered as a consequence of (4.3) and the arguments symmetry property

$$
R_{12}^{\hbar}(z)=R_{12}^{\frac{z}{N}}(N \hbar) P_{12},
$$

which follows from (A.12)-( $(\mathrm{A.13})$. In order to verify (4.4) directly consider

$$
\left(T_{\gamma}\right)_{1}^{-1} R_{12}^{\hbar}(z)\left(T_{\gamma}\right)_{2}
$$

for some fixed $T_{\gamma}$ (A.17). Here the lower indices 1,2 are the numbers of the tensor components (4.2), and $T_{\gamma}^{-1}=T_{-\gamma}$ due to (A.18). For the above expression we have:

$$
=\sum_{\alpha \in \mathbb{Z}_{N} \times \mathbb{Z}_{N}} \varphi_{\alpha}\left(z, \omega_{\alpha}+\hbar\right) T_{-\gamma} T_{\alpha} \otimes T_{-\alpha} T_{\gamma}=\sum_{\alpha \in \mathbb{Z}_{N} \times \mathbb{Z}_{N}} \varphi_{\alpha}\left(z, \omega_{\alpha}+\hbar\right) T_{\alpha-\gamma} \otimes T_{\gamma-\alpha}
$$

Now shift the summation indices $\alpha \rightarrow \alpha+\gamma$. Then from (A.22) we get

$$
\left(T_{\gamma}\right)_{1}^{-1} R_{12}^{\hbar}(z)\left(T_{\gamma}\right)_{2}=\exp \left(2 \pi \imath \partial_{\tau} \omega_{\gamma}\right) R_{12}^{\hbar+\omega_{\gamma}}(z) .
$$

This answer reproduces (4.4) for $\gamma=(1,0)$ and $\gamma=(0,1)$ (then $T_{\gamma}=Q, \omega_{\gamma}=1 / N$ and $T_{\gamma}=\Lambda$, $\omega_{\gamma}=\tau / N$ respectively). 
Notice that in the proof of (4.4) we used the shift of summation indices $(\alpha \rightarrow \alpha+\gamma)$. At the same time the limits of the sum were not changed because $\alpha \in \mathbb{Z}_{N} \times \mathbb{Z}_{N}$. In fact, we also used the periodicity of functions (A.10) $\varphi_{\alpha}\left(z, \omega_{\alpha}+\hbar\right)$ in indices considered as discrete variables:

$$
\varphi_{\alpha_{1}+N, \alpha_{2}}\left(z, \omega_{\alpha}+\omega_{(N, 0)}+\hbar\right)=\varphi_{\alpha_{1}, \alpha_{2}+N}\left(z, \omega_{\alpha}+\omega_{(0, N)}+\hbar\right)=\varphi_{\alpha}\left(z, \omega_{\alpha}+\hbar\right) .
$$

The property (4.7) demonstrates necessity of the exponential factor $\exp \left(2 \pi \imath \partial_{\tau} \omega_{\alpha}\right)$ in the definition (A.10).

Symmetric $R$-matrix in $\operatorname{Mat}(N, \mathbb{C})^{\otimes 2} \otimes \operatorname{Mat}(M, \mathbb{C})^{\otimes 2}$. In this section we assume that $N$ and $M$ are coprime integers unless otherwise specified 4 .

Consider the following expression:

$$
\Re_{12, \tilde{1} \tilde{2}}(z, \hbar)=\sum_{\alpha \in \mathbb{Z}_{N} \times \mathbb{Z}_{N}} \sum_{\tilde{\alpha} \in \mathbb{Z}_{M} \times \mathbb{Z}_{M}} \Phi_{\alpha, \tilde{\alpha}}(z, \hbar) T_{\alpha} \otimes T_{-\alpha} \otimes \tilde{T}_{\tilde{\alpha}} \otimes \tilde{T}_{-\tilde{\alpha}},
$$

where (with the definition (A.10)

$$
\begin{gathered}
\Phi_{\alpha, \tilde{\alpha}}(z, \hbar)=\exp \left(2 \pi \imath\left(z+N \tilde{\omega}_{\tilde{\alpha}}\right) \frac{\alpha_{2}}{N}+2 \pi \imath \hbar N \frac{\tilde{\alpha}_{2}}{M}\right) \phi\left(z+N \tilde{\omega}_{\tilde{\alpha}}, \hbar+\omega_{\alpha}\right)= \\
=\exp \left(2 \pi \imath \hbar N \frac{\tilde{\alpha}_{2}}{M}\right) \varphi_{\alpha}\left(z+N \tilde{\omega}_{\tilde{\alpha}}, \hbar+\omega_{\alpha}\right)
\end{gathered}
$$

and for any $\alpha=\left(\alpha_{1}, \alpha_{2}\right) \in \mathbb{Z}_{N} \times \mathbb{Z}_{N}$ and $\tilde{\alpha}=\left(\tilde{\alpha}_{1}, \tilde{\alpha}_{2}\right) \in \mathbb{Z}_{M} \times \mathbb{Z}_{M}$

$$
\omega_{\alpha}=\frac{\alpha_{1}+\alpha_{2} \tau}{N}, \quad \tilde{\omega}_{\tilde{\alpha}}=\frac{\tilde{\alpha}_{1}+\tilde{\alpha}_{2} \tau}{M}
$$

while $\left\{T_{\alpha}\right\}$ (and $\left.\left\{\tilde{T}_{\tilde{\alpha}}\right\}\right)$ is the basis in $\operatorname{Mat}(N, \mathbb{C})$ (and $\operatorname{Mat}(M, \mathbb{C})$ respectively) defined as in (A.14)-(A.18). That is, we mark the $\operatorname{Mat}(M, \mathbb{C})$ elements and related quantities (including related indices) by tildes. For example, $\tilde{\kappa}_{\tilde{\alpha}, \tilde{\beta}}=\exp \left(\pi \imath\left(\tilde{\beta}_{1} \tilde{\alpha}_{2}-\tilde{\beta}_{2} \tilde{\alpha}_{1}\right) / M\right)$. For coprime $N$ and $M$ two sets $\left\{f\left(N \tilde{\omega}_{\tilde{\alpha}}\right), \tilde{\alpha} \in \mathbb{Z}_{M} \times \mathbb{Z}_{M}\right\}$ and $\left\{f\left(\tilde{\omega}_{\tilde{\alpha}}\right), \tilde{\alpha} \in \mathbb{Z}_{M} \times \mathbb{Z}_{M}\right\}$ are equal for a periodic function $f$ on the lattice $\mathbb{Z}_{M} \times \mathbb{Z}_{M}$.

First, notice that for $M=1$ (then $\tilde{\alpha}=(0,0)$ ) expression (4.8) reproduces the definition of the Belavin's $\operatorname{Mat}(N, \mathbb{C}) R$-matrix (1.6) $R_{12}^{\hbar}(z) \in \operatorname{Mat}(N, \mathbb{C})^{\otimes 2}$, and similarly for $N=1$ we have $R_{12}^{z}(\hbar) \in \operatorname{Mat}(M, \mathbb{C})^{\otimes 2}$.

The analogues of the quasiperiodic properties (boundary conditions) on the lattice $\mathbb{Z} \oplus \tau \mathbb{Z}$ are of the form:

$$
\begin{gathered}
\mathfrak{R}_{12, \tilde{1} \tilde{2}}(z+1, \hbar)=Q_{1}^{-1} \mathfrak{R}_{12, \tilde{1} \tilde{2}}(z, \hbar) Q_{1}, \\
\mathfrak{R}_{12, \tilde{1} \tilde{2}}(z+\tau, \hbar)=\exp (-2 \pi \imath \hbar) \Lambda_{1}^{-1} \mathfrak{R}_{12, \tilde{1} \tilde{2}}(z, \hbar) \Lambda_{1}
\end{gathered}
$$

and

$$
\begin{gathered}
\mathfrak{R}_{12, \tilde{1} \tilde{2}}(z, \hbar+1)=\tilde{Q}_{\tilde{1}}^{-N} \Re_{12, \tilde{1} \tilde{2}}(z, \hbar) \tilde{Q}_{\tilde{1}}^{N}, \\
\mathfrak{R}_{12, \tilde{1} \tilde{2}}(z, \hbar+\tau)=\exp (-2 \pi \imath z) \tilde{\Lambda}_{\tilde{1}}^{-N} \mathfrak{R}_{12, \tilde{1} \tilde{2}}(z, \hbar) \tilde{\Lambda}_{\tilde{1}}^{N},
\end{gathered}
$$

\footnotetext{
${ }^{4}$ The general case is not difficult but requires more additional notations. We consider special case $M=N$ in the next section.
} 
where $Q_{1}=Q \otimes 1_{N} \otimes \tilde{1}_{M} \otimes \tilde{1}_{M}, \tilde{Q}_{\tilde{1}}=1_{N} \otimes 1_{N} \otimes \tilde{Q} \otimes \tilde{1}_{M}$, and $\tilde{Q}, \tilde{\Lambda}$ are the matrices of form (A.14) but of size $M \times M$.

It is important for us that functions $\Phi_{\alpha, \tilde{\alpha}}(z, \hbar)$ are periodic in both discrete variables - indices $\alpha=\left(\alpha_{1}, \alpha_{2}\right) \in \mathbb{Z}_{N} \times \mathbb{Z}_{N}$ and $\tilde{\alpha}=\left(\tilde{\alpha}_{1}, \tilde{\alpha}_{2}\right) \in \mathbb{Z}_{M} \times \mathbb{Z}_{M}$, i.e. similarly to (4.7) we have

$$
\Phi_{\left(\alpha_{1}+N, \alpha_{2}\right), \tilde{\alpha}}(z, \hbar)=\Phi_{\left(\alpha_{1}, \alpha_{2}+N\right), \tilde{\alpha}}(z, \hbar)=\Phi_{\left(\alpha_{1}, \alpha_{2}\right), \tilde{\alpha}}(z, \hbar)
$$

and

$$
\Phi_{\alpha,\left(\tilde{\alpha}_{1}+M, \tilde{\alpha}_{2}\right)}(z, \hbar)=\Phi_{\alpha,\left(\tilde{\alpha}_{1}, \tilde{\alpha}_{2}+M\right)}(z, \hbar)=\Phi_{\alpha,\left(\tilde{\alpha}_{1}, \tilde{\alpha}_{2}\right)}(z, \hbar) .
$$

These properties allow to shift indices of summations in the same way as it was used in (4.6). To verify (4.11)-(4.12) and (4.13)-(4.14) one needs (4.4).

In $\mathrm{GL}_{N}$ case we had also quasiperiodic behavior (4.4) on the smaller elliptic curve with periods $1 / N$ and $\tau / N$. For the symmetric $R$-matrix (4.4) acquires the form:

$$
\begin{gathered}
\mathfrak{R}_{12, \tilde{1} \tilde{2}}\left(z+N \frac{1}{M}, \hbar\right)=\tilde{Q}_{\tilde{1}}^{-1} \Re_{12, \tilde{1} \tilde{2}}(z, \hbar) \tilde{Q}_{\tilde{2}} \\
\mathfrak{R}_{12, \tilde{1} \tilde{2}}\left(z+N \frac{\tau}{M}, \hbar\right)=\exp \left(-2 \pi \imath N \frac{\hbar}{M}\right) \tilde{\Lambda}_{\tilde{1}}^{-1} \mathfrak{R}_{12, \tilde{1} \tilde{2}}(z, \hbar) \tilde{\Lambda}_{\tilde{2}}
\end{gathered}
$$

and

$$
\begin{gathered}
\Re_{12, \tilde{1} \tilde{2}}\left(z, \hbar+\frac{1}{N}\right)=Q_{1}^{-1} \tilde{Q}_{\tilde{1}}^{-1} \mathfrak{R}_{12, \tilde{1} \tilde{2}}(z, \hbar) \tilde{Q}_{\tilde{1}} Q_{2}, \\
\mathfrak{R}_{12, \tilde{1} \tilde{2}}\left(z, \hbar+\frac{\tau}{N}\right)=\exp \left(-2 \pi \imath \frac{z}{N}\right) \Lambda_{1}^{-1} \tilde{\Lambda}_{\tilde{1}}^{-1} \mathfrak{R}_{12, \tilde{1} \tilde{2}}(z, \hbar) \tilde{\Lambda}_{\tilde{1}} \Lambda_{2} .
\end{gathered}
$$

The proof of (4.15)-(4.16) is similar to the one given for (4.7). For example, to get (4.16) consider

$$
\left(T_{\gamma}\right)_{1}^{-1} \Re_{12, \tilde{1} \tilde{2}}(z, \hbar)\left(T_{\gamma}\right)_{2}=\sum_{\alpha \in \mathbb{Z}_{N} \times \mathbb{Z}_{N}} \sum_{\tilde{\alpha} \in \mathbb{Z}_{M} \times \mathbb{Z}_{M}} \Phi_{\alpha, \tilde{\alpha}}(z, \hbar) T_{\alpha-\gamma} \otimes T_{\gamma-\alpha} \otimes \tilde{T}_{\tilde{\alpha}} \otimes \tilde{T}_{-\tilde{\alpha}} .
$$

Then, shift the summation index as $\alpha \rightarrow \alpha+\gamma$ and notice that

$$
\Phi_{\alpha+\gamma, \tilde{\alpha}}(z, \hbar)=\exp \left(2 \pi \imath z \partial_{\tau} \omega_{\gamma}\right) \tilde{\kappa}_{\gamma, \tilde{\alpha}}^{2} \Phi_{\alpha, \tilde{\alpha}}\left(z, \hbar+\omega_{\gamma}\right)
$$

Plugging $\gamma=(1,0)$ (or $\gamma=(0,1))$ into the obtained expression one gets the upper (or the lower) line of (4.16).

Quadratic relations. Let us prove that the symmetric $R$-matrix obeys the relations similar to (1.1), (1.7) and (1.8).

Proposition 4.1 The symmetric R-matrix (4.8) satisfies the following set of properties:

1. Skew-symmetry:

$$
\mathfrak{R}_{21, \tilde{2} \tilde{1}}(-z,-\hbar)=-\mathfrak{R}_{12, \tilde{1} \tilde{2}}(z, \hbar)
$$

2. Associative Yang-Baxter equation:

$$
\mathfrak{R}_{12, \tilde{1} \tilde{2}} \mathfrak{R}_{23, \tilde{3} \tilde{2}}=\mathfrak{R}_{13, \tilde{3} \tilde{2}} \mathfrak{R}_{12, \tilde{1} \tilde{3}}+\mathfrak{R}_{23, \tilde{3} \tilde{1}} \mathfrak{R}_{13, \tilde{1} \tilde{2}}
$$

where $\mathfrak{R}_{a b, \tilde{a} \tilde{b}}=\mathfrak{R}_{a b, \tilde{a} \tilde{b}}\left(z_{a}-z_{b}, \hbar_{\tilde{a}}-\hbar_{\tilde{b}}\right)$. 


\section{Unitarity:}

$$
\Re_{12, \tilde{1} \tilde{2}} \Re_{21, \tilde{1} \tilde{2}}=1_{N} \otimes 1_{N} \otimes \tilde{1}_{M} \otimes \tilde{1}_{M} N^{2} M^{2}(\wp(N \hbar)-\wp(M z))
$$

or

$$
\mathfrak{R}_{12, \tilde{1} \tilde{2}} \mathfrak{R}_{12, \tilde{2} \tilde{1}}=1_{N} \otimes 1_{N} \otimes \tilde{1}_{M} \otimes \tilde{1}_{M} N^{2} M^{2}(\wp(M z)-\wp(N \hbar))
$$

Proof: The first property simply follows from the definition (4.8)-(4.9):

$$
\mathfrak{R}_{21, \tilde{2} \tilde{1}}(-z,-\hbar)=\sum_{\alpha \in \mathbb{Z}_{N} \times \mathbb{Z}_{N}} \sum_{\tilde{\alpha} \in \mathbb{Z}_{M} \times \mathbb{Z}_{M}} \Phi_{\alpha, \tilde{\alpha}}(-z,-\hbar) T_{-\alpha} \otimes T_{\alpha} \otimes \tilde{T}_{-\tilde{\alpha}} \otimes \tilde{T}_{\tilde{\alpha}}
$$

Changing summation indices as $\alpha \rightarrow-\alpha, \tilde{\alpha} \rightarrow-\tilde{\alpha}$ and using $\Phi_{-\alpha,-\tilde{\alpha}}(-z,-\hbar)=-\Phi_{\alpha, \tilde{\alpha}}(z, \hbar)$ we get (4.17).

The second property follows from (A.19) (for both $\kappa$ and $\tilde{\kappa}$ ) and the Fay identity (A.5) written in terms functions (4.9) as

$$
\begin{aligned}
& \Phi_{\alpha, \tilde{\alpha}}(z, \hbar) \Phi_{\beta, \tilde{\beta}}(w, \eta)= \\
& =\Phi_{\beta, \tilde{\alpha}+\tilde{\beta}}(z+w, \eta) \Phi_{\alpha-\beta, \tilde{\alpha}}(z, \hbar-\eta)+\Phi_{\beta-\alpha, \tilde{\beta}}(w, \eta-\hbar) \Phi_{\alpha, \tilde{\alpha}+\tilde{\beta}}(z+w, \hbar)
\end{aligned}
$$

with $z=z_{1}-z_{2}, w=z_{2}-z_{3}, \hbar=\hbar_{\tilde{1}}-\hbar_{\tilde{2}}$ and $\eta=\hbar_{\tilde{3}}-\hbar_{\tilde{2}}$.

To prove the unitarity (4.19) let us write its l.h.s. explicitly

$$
\Re_{12, \tilde{1} \tilde{2}}(z, \hbar) \Re_{21, \tilde{1} \tilde{2}}(-z, \hbar)=\sum_{\alpha, \beta, \tilde{\alpha}, \tilde{\beta}} \Phi_{\alpha, \tilde{\alpha}}(z, \hbar) \Phi_{\beta, \tilde{\beta}}(-z, \hbar) \kappa_{\alpha,-\beta}^{2} \tilde{\kappa}_{\tilde{\alpha}, \tilde{\beta}}^{2} T_{\alpha-\beta} \otimes T_{\beta-\alpha} \otimes \tilde{T}_{\tilde{\alpha}+\tilde{\beta}} \otimes \tilde{T}_{-\tilde{\alpha}-\tilde{\beta}}
$$

and subdivide this sum into four parts as

$$
\sum_{\alpha, \beta, \tilde{\alpha}, \tilde{\beta}}=\sum_{\alpha=\beta, \tilde{\alpha}=-\tilde{\beta}}+\sum_{\alpha \neq \beta, \tilde{\alpha}=-\tilde{\beta}}+\sum_{\alpha=\beta, \tilde{\alpha} \neq-\tilde{\beta}}+\sum_{\alpha \neq \beta, \tilde{\alpha} \neq-\tilde{\beta}}
$$

The first sum reproduces the answer due to (A.7) and (A.11) 5 :

$\sum_{\alpha=\beta, \tilde{\alpha}=-\tilde{\beta}} \Phi_{\alpha, \tilde{\alpha}}(z, \hbar) \Phi_{\beta, \tilde{\beta}}(-z, \hbar)=\sum_{\alpha} \sum_{\tilde{\alpha}} \wp\left(\hbar+\omega_{\alpha}\right)-\wp\left(z+N \tilde{\omega}_{\alpha}\right)=N^{2} M^{2}(\wp(N \hbar)-\wp(M z))$.

Each of three other sums equals zero. The vanishing of the second and the third sum is proved in the same way as it is made for unitarity of $R^{\hbar}(z)$ in the Appendix. Consider the fourth sum. Denote $\gamma=\alpha-\beta, \tilde{\gamma}=\tilde{\alpha}+\tilde{\beta}$. Then, using (4.21)

$$
\begin{gathered}
\sum_{\alpha \neq \beta, \tilde{\alpha} \neq-\tilde{\beta}}=\sum_{\gamma \neq 0, \tilde{\gamma} \neq 0} T_{\gamma} \otimes T_{-\gamma} \otimes \tilde{T}_{\tilde{\gamma}} \otimes \tilde{T}_{-\tilde{\gamma}} \times \\
\times\left(\sum_{\beta, \tilde{\alpha}} \kappa_{\beta, \gamma}^{2} \tilde{\kappa}_{\tilde{\alpha}, \tilde{\gamma}}^{2} \Phi_{\beta, \tilde{\gamma}}(0, \hbar) \Phi_{\gamma, \tilde{\alpha}}(z, 0)+\sum_{\alpha, \tilde{\beta}} \kappa_{\alpha, \gamma}^{2} \tilde{\kappa}_{\tilde{\gamma}, \tilde{\beta}}^{2} \Phi_{\alpha, \tilde{\gamma}}(0, \hbar) \Phi_{-\gamma, \tilde{\beta}}(-z, 0)\right) .
\end{gathered}
$$

\footnotetext{
${ }^{5}$ We also use here that $\sum_{\tilde{\alpha}} \wp\left(z+N \tilde{\omega}_{\alpha}\right)=\sum_{\tilde{\alpha}} \wp\left(z+\tilde{\omega}_{\alpha}\right)$ for coprime $N$ and $M$.
} 
The latter two terms cancel each other after changing notation $\alpha:=\beta, \tilde{\beta}:=-\tilde{\alpha}$ in the last term. This finishes the proof of (4.19). At last, (4.20) follows from (4.19) and (4.17).

Cubic relations. The next step is to derive cubic relations of (2.1)-(2.4) type. Recall that (2.3) is the most general in that list. In a similar way one can obtain its analogue for the symmetric $R$-matrix (4.8). First, multiply (4.18) by $\mathfrak{R}_{23, \tilde{1} \tilde{3}}$ from the left:

$$
\mathfrak{R}_{23, \tilde{1} \tilde{3}} \mathfrak{R}_{12, \tilde{1} \tilde{2}} \mathfrak{R}_{23, \tilde{3} \tilde{2}}=\mathfrak{R}_{23, \tilde{1} \tilde{3}} \mathfrak{R}_{13, \tilde{3} \tilde{2}} \mathfrak{R}_{12, \tilde{1} \tilde{3}}+\mathfrak{R}_{23, \tilde{1} \tilde{3}} \mathfrak{R}_{23, \tilde{3} \tilde{1}} \mathfrak{R}_{13, \tilde{1} \tilde{2}}
$$

Second, change indices $2 \leftrightarrow 3$ in (4.18) and multiply it by $\mathfrak{R}_{23, \tilde{3} \tilde{2}}$ from the right:

$$
\mathfrak{R}_{13, \tilde{1} \tilde{2} \tilde{}} \mathfrak{R}_{32, \tilde{3} \tilde{2}} \mathfrak{R}_{23, \tilde{3} \tilde{2}}=\mathfrak{R}_{12, \tilde{3} \tilde{2}} \mathfrak{R}_{13, \tilde{1} \tilde{3}} \mathfrak{R}_{23, \tilde{3} \tilde{2}}+\mathfrak{R}_{32, \tilde{3} \tilde{1} \tilde{}} \mathfrak{R}_{12, \tilde{1} \tilde{2}} \mathfrak{R}_{23, \tilde{3} \tilde{2}}
$$

Combining these two equations and using the properties of skew-symmetry (4.17) and unitarity (4.19)-(4.20) we get

$$
\mathfrak{R}_{12, \tilde{3} \tilde{2}} \mathfrak{R}_{13, \tilde{1} \tilde{3}} \mathfrak{R}_{23, \tilde{3} \tilde{2}}=\mathfrak{R}_{23, \tilde{1} \tilde{3}} \mathfrak{R}_{13, \tilde{3} \tilde{2}} \mathfrak{R}_{12, \tilde{1} \tilde{3}}+N^{2} M^{2} \mathfrak{R}_{13, \tilde{1} \tilde{2}}\left(\wp\left(N \hbar_{\tilde{3} \tilde{2}}\right)-\wp\left(N \hbar_{\tilde{1} \tilde{3}}\right)\right),
$$

where $\hbar_{\tilde{a} \tilde{b}}=\hbar_{\tilde{a}}-\hbar_{\tilde{b}}$. When $M=1$ (4.22) coincides with (2.3), where $\hbar=\hbar_{\tilde{1}}-\hbar_{\tilde{3}}$ and $\eta=\hbar_{\tilde{3}}-\hbar_{\tilde{2}}$.

In particular case of $\hbar_{\tilde{3}}=\left(\hbar_{\tilde{1}}+\hbar_{\tilde{2}}\right) / 2$ we get Yang-Baxter like equation

$$
\mathfrak{R}_{12, \tilde{3} \tilde{2}}\left(z_{12}, \hbar\right) \mathfrak{R}_{13, \tilde{1} \tilde{3}}\left(z_{13}, \hbar\right) \mathfrak{R}_{23, \tilde{3} \tilde{2}}\left(z_{23}, \hbar\right)=\mathfrak{R}_{23, \tilde{1} \tilde{3}}\left(z_{23}, \hbar\right) \mathfrak{R}_{13, \tilde{3} \tilde{2}}\left(z_{13}, \hbar\right) \mathfrak{R}_{12, \tilde{1} \tilde{3}}\left(z_{12}, \hbar\right)
$$

with $\hbar=\tilde{\hbar}_{\tilde{1} \tilde{3}}=\tilde{\hbar}_{\tilde{3} \tilde{2}}$.

Another particular case $\tilde{\hbar}_{\tilde{1}}=\tilde{\hbar}_{\tilde{2}}$ in (4.22) leads to analogue of (2.2):

$$
\begin{gathered}
\Re_{21, \tilde{2} \tilde{3}}\left(z_{21}, \hbar\right) \mathfrak{R}_{13, \tilde{1} \tilde{3}}\left(z_{13}, \hbar\right) \Re_{32, \tilde{2} \tilde{3}}\left(z_{32}, \hbar\right)+\mathfrak{R}_{23, \tilde{1} \tilde{3}}\left(z_{23}, \hbar\right) \mathfrak{R}_{31, \tilde{2} \tilde{3}}\left(z_{31}, \hbar\right) \mathfrak{R}_{12, \tilde{1} \tilde{3}}\left(z_{12}, \hbar\right)= \\
=-1_{N} \otimes 1_{N} \otimes \tilde{P}_{\tilde{1} \tilde{3}} N^{3} M^{2} \wp^{\prime}(N \hbar) .
\end{gathered}
$$

A similar derivation leads to the cubic equation of (4.22) type with $\hbar$ and $z$ variables interchanged:

$$
\mathfrak{R}_{32, \tilde{1} \tilde{2}} \mathfrak{R}_{13, \tilde{1} \tilde{3}} \mathfrak{R}_{32, \tilde{2} \tilde{3}}=\mathfrak{R}_{13, \tilde{2} \tilde{3}} \mathfrak{R}_{32, \tilde{1} \tilde{3}} \mathfrak{R}_{13, \tilde{1} \tilde{2}}+N^{2} M^{2} \mathfrak{R}_{12, \tilde{1} \tilde{3}}\left(\wp\left(M z_{23}\right)-\wp\left(M z_{13}\right)\right),
$$

where $z_{a b}=z_{a}-z_{b}$.

Now we can easily obtain the cubic Yang-Baxter like equations of (2.4) type. It is based on the skew-symmetry of $\wp\left(N \hbar_{\tilde{3} \tilde{2}}\right)-\wp\left(N \hbar_{\tilde{1} \tilde{3}}\right)$ with respect to interchanging of the arguments only. Therefore, from (4.22) we have:

$$
\begin{aligned}
& \mathfrak{R}_{12, \tilde{3} \tilde{2}}\left(z_{12}, \hbar_{\tilde{3} \tilde{2}}\right) \mathfrak{R}_{13, \tilde{1} \tilde{3}}\left(z_{13}, \hbar_{\tilde{1} \tilde{3}}\right) \mathfrak{R}_{23, \tilde{3} \tilde{2}}\left(z_{23}, \hbar_{\tilde{3} \tilde{2}}\right)+\mathfrak{R}_{12, \tilde{3} \tilde{2}}\left(z_{12}, \hbar_{\tilde{1} \tilde{3}}\right) \mathfrak{R}_{13, \tilde{1} \tilde{3}}\left(z_{13}, \hbar_{\tilde{3} \tilde{2}}\right) \mathfrak{R}_{23, \tilde{3} \tilde{2}}\left(z_{23}, \hbar_{\tilde{1} \tilde{3}}\right) \\
& =\mathfrak{R}_{23, \tilde{1} \tilde{3}}\left(z_{23}, \hbar_{1 \tilde{3}}\right) \mathfrak{R}_{13, \tilde{3} \tilde{2}}\left(z_{13}, \hbar_{\tilde{3} \tilde{2}}\right) \mathfrak{R}_{12, \tilde{1} \tilde{3}}\left(z_{12}, \hbar_{\tilde{1} \tilde{3}}\right)+\mathfrak{R}_{23, \tilde{1} \tilde{3}}\left(z_{23}, \hbar_{\tilde{3} \tilde{2}}\right) \mathfrak{R}_{13, \tilde{3} \tilde{2}}\left(z_{13}, \hbar_{\tilde{1} \tilde{3}}\right) \mathfrak{R}_{12, \tilde{1} \tilde{3}}\left(z_{12}, \hbar_{\tilde{3} \tilde{2}}\right)
\end{aligned}
$$

In the same manner from (4.25) we get:

$$
\begin{aligned}
& \mathfrak{R}_{32, \tilde{1} \tilde{2}}\left(z_{32}, \hbar_{\tilde{1} \tilde{2}}\right) \mathfrak{R}_{13, \tilde{1} \tilde{3}}\left(z_{13}, \hbar_{\tilde{1} \tilde{3}}\right) \mathfrak{R}_{32, \tilde{2} \tilde{3}}\left(z_{32}, \hbar_{\tilde{2} \tilde{3}}\right)+\mathfrak{R}_{32, \tilde{1} \tilde{2}}\left(z_{13}, \hbar_{\tilde{1} \tilde{2}}\right) \mathfrak{R}_{32, \tilde{1} \tilde{3}}\left(z_{13}, \hbar_{\tilde{1} \tilde{3}}\right) \mathfrak{R}_{32, \tilde{2} \tilde{3}}\left(z_{13}, \hbar_{\tilde{2} \tilde{3}}\right) \\
& =\mathfrak{R}_{13, \tilde{2} \tilde{3}}\left(z_{13}, \hbar_{\tilde{2} \tilde{3}}\right) \mathfrak{R}_{32, \tilde{1} \tilde{3}}\left(z_{32}, \hbar_{\tilde{1} \tilde{3}}\right) \mathfrak{R}_{13, \tilde{1} \tilde{2}}\left(z_{13}, \hbar_{\tilde{1} \tilde{2}}\right)+\mathfrak{R}_{13, \tilde{2} \tilde{3}}\left(z_{32}, \hbar_{\tilde{2} \tilde{3}}\right) \mathfrak{R}_{32, \tilde{1} \tilde{3}}\left(z_{13}, \hbar_{\tilde{1} \tilde{3}}\right) \mathfrak{R}_{13, \tilde{1} \tilde{2}}\left(z_{32}, \hbar_{\tilde{1} \tilde{2}}\right)
\end{aligned}
$$


Relation to Baxter-Belavin $\mathrm{GL}_{N M} R$-matrix. Multiply the symmetric $R$-matrix by $N P_{12} \otimes$ $\tilde{1}_{M} \otimes \tilde{1}_{M}$ :

$$
\begin{gathered}
\Re_{12, \tilde{1} \tilde{2}}(z, \hbar) N P_{12} \otimes \tilde{1}_{M} \otimes \tilde{1}_{M}=\sum_{\alpha, \beta \in \mathbb{Z}_{N} \times \mathbb{Z}_{N}} \sum_{\tilde{\alpha} \in \mathbb{Z}_{M} \times \mathbb{Z}_{M}} \Phi_{\alpha, \tilde{\alpha}}(z, \hbar) T_{\alpha} T_{\beta} \otimes T_{-\alpha} T_{-\beta} \otimes \tilde{T}_{\tilde{\alpha}} \otimes \tilde{T}_{-\tilde{\alpha}} \\
=\sum_{\alpha, \gamma, \tilde{\alpha}} \kappa_{\alpha, \gamma}^{2} \exp \left(N \hbar \partial_{\tau} \tilde{\omega}_{\tilde{\alpha}}\right) \varphi_{\alpha}\left(z+N \tilde{\omega}_{\tilde{\alpha}}, \hbar+\omega_{\alpha}\right) T_{\gamma} \otimes T_{-\gamma} \otimes \tilde{T}_{\tilde{\alpha}} \otimes \tilde{T}_{-\tilde{\alpha}} \underline{=A .13} \\
=N \sum_{\gamma, \tilde{\alpha}} \varphi_{\gamma+\tilde{\alpha}}\left(N \hbar, \omega_{\gamma}+\tilde{\omega}_{\tilde{\alpha}}+\frac{z}{N}\right) T_{\gamma} \otimes T_{-\gamma} \otimes \tilde{T}_{\tilde{\alpha}} \otimes \tilde{T}_{-\tilde{\alpha}} .
\end{gathered}
$$

Since $M$ and $N$ are coprime the summation over $\omega_{\gamma}+\tilde{\omega}_{\tilde{\alpha}}$ is equivalent to the summation over $\omega_{a}$, where $a \in \mathbb{Z}_{M N} \times \mathbb{Z}_{M N}$. Therefore, we get the expression which is the GL TM $_{M}$ Baxter-Belavin's $R$-matrix written in special basis.

Particular case $M=N$. In this particular case the function $\Phi_{\alpha, \tilde{\alpha}}(z, \hbar)$ (4.9) is simplified. Using (A.4) it is easy to get that

$$
\Phi_{\alpha, \tilde{\alpha}}(z, \hbar)=\exp \left(2 \pi \imath \frac{\tilde{\alpha}_{1} \alpha_{2}-\tilde{\alpha}_{2} \alpha_{1}}{N}\right) \varphi_{\alpha}\left(z, \omega_{\alpha}+\hbar\right)=\kappa_{\alpha, \tilde{\alpha}}^{2} \varphi_{\alpha}\left(z, \omega_{\alpha}+\hbar\right) .
$$

\section{Appendix}

\subsection{Elliptic functions}

We deal with the following elliptic functions:

$$
\begin{gathered}
\phi(z, u)=\frac{\vartheta^{\prime}(0) \vartheta(z+u)}{\vartheta(z) \vartheta(u)}, \\
E_{1}(z)=\frac{\vartheta^{\prime}(z)}{\vartheta(z)}, \quad E_{2}(z)=-\partial_{z} E_{1}(z)=\wp(z)-\frac{1}{3} \frac{\vartheta^{\prime \prime \prime}(0)}{\vartheta^{\prime}(0)},
\end{gathered}
$$

where $\vartheta(z)$ is the Riemann theta-function

$$
\vartheta(z)=\vartheta(z \mid \tau)=\sum_{k \in \mathbb{Z}} \exp \left(\pi \imath \tau\left(k+\frac{1}{2}\right)^{2}+2 \pi \imath\left(z+\frac{1}{2}\right)\left(k+\frac{1}{2}\right)\right)
$$

which has simple zero at $z=0$, and $\wp(z)$ in (A.2) is the Weierstrass $\wp$-function.

The function $\phi(x, y)=\phi(y, x)$ has the quasiperiodic properties

$$
\phi(x+1, y)=\phi(x, y), \quad \phi(x+\tau, y)=\exp (-2 \pi \imath y) \phi(x, y)
$$

and satisfies the Fay type identities:

$$
\begin{gathered}
\phi(x, u) \phi(y, w)=\phi(x-y, u) \phi(y, u+w)+\phi(y-x, w) \phi(x, u+w), \\
\phi(x, z) \phi(x, w)=\phi(x, z+w)\left(E_{1}(x)+E_{1}(z)+E_{1}(w)-E_{1}(x+z+w)\right), \\
\phi(z, u) \phi(z,-u)=\wp(z)-\wp(u)=E_{2}(z)-E_{2}(u) .
\end{gathered}
$$


These relations lead to

$$
\begin{gathered}
\phi\left(z, u_{1}-v\right) \phi\left(w, u_{2}+v\right) \phi(z-w, v)-\phi\left(z, u_{2}+v\right) \phi\left(w, u_{1}-v\right) \phi\left(z-w, u_{1}-u_{2}-v\right) \\
=\phi\left(z, u_{1}\right) \phi\left(w, u_{2}\right)\left(E_{1}(v)-E_{1}\left(u_{1}-u_{2}-v\right)+E_{1}\left(u_{1}-v\right)-E_{1}\left(u_{2}+v\right)\right)
\end{gathered}
$$

and

$$
\begin{gathered}
\phi(z, u-v) \phi(w, v) \phi(z-w, v)-\phi(z, v) \phi(w, u-v) \phi(z-w, u-v) \\
=\phi(z, u)(\wp(v)-\wp(u-v)) .
\end{gathered}
$$

We also need the following set of functions:

$$
\varphi_{\alpha}\left(z, \omega_{\alpha}+\hbar\right)=\exp \left(2 \pi \imath \frac{\alpha_{2}}{N} z\right) \phi\left(z, \omega_{\alpha}+\hbar\right), \quad \alpha \in \mathbb{Z}^{\times 2} .
$$

These functions satisfy the set of identities (A.5)-(A.9) because the exponential factors are canceled.

Averaging $\wp$-function:

$$
\sum_{\alpha \in \mathbb{Z}_{N} \times \mathbb{Z}_{N}} \wp\left(\omega_{\alpha}+\hbar\right)=N^{2} \wp(N \hbar)
$$

The following formulae (of finite Fourier transformation type) are also useful in derivation of $R$-matrix identities (see [14]):

$$
\frac{1}{N} \sum_{\alpha \in \mathbb{Z}_{N} \times \mathbb{Z}_{N}} \kappa_{\alpha, \gamma}^{2} \varphi_{\alpha}\left(N \hbar, \omega_{\alpha}+\frac{z}{N}\right)=\varphi_{\gamma}\left(z, \omega_{\gamma}+\hbar\right), \quad \forall \gamma \in \mathbb{Z}^{\times 2}
$$

or (exchanging the arguments)

$$
\frac{1}{N} \sum_{\alpha \in \mathbb{Z}_{N} \times \mathbb{Z}_{N}} \kappa_{\alpha, \gamma}^{2} \varphi_{\alpha}\left(z, \omega_{\alpha}+\hbar\right)=\varphi_{\gamma}\left(N \hbar, \omega_{\gamma}+\frac{z}{N}\right), \quad \forall \gamma \in \mathbb{Z}^{\times 2},
$$

where $\kappa_{\alpha, \beta}$ is from (A.18).

\subsection{Baxter-Belavin $R$-matrix}

Finite-dimensional representation of the Heisenberg group. Consider the following $N \times N$ matrices:

$$
\begin{gathered}
Q, \Lambda \in \operatorname{Mat}(N, \mathbb{C}): \quad Q_{k l}=\delta_{k l} \exp \left(\frac{2 \pi \imath}{N} k\right), \quad \Lambda_{k l}=\delta_{k-l+1=0 \bmod N}, \quad k, l=1, \ldots, N, \\
Q^{N}=\Lambda^{N}=1, \\
\exp \left(2 \pi \imath \frac{\gamma_{1} \gamma_{2}}{N}\right) Q^{\gamma_{1}} \Lambda^{\gamma_{2}}=\Lambda^{\gamma_{2}} Q^{\gamma_{1}}, \quad \gamma_{1}, \gamma_{2} \in \mathbb{Z} .
\end{gathered}
$$

Introduce

$$
T_{\gamma}:=T_{\gamma_{1} \gamma_{2}}=\exp \left(\pi \imath \frac{\gamma_{1} \gamma_{2}}{N}\right) Q^{\gamma_{1}} \Lambda^{\gamma_{2}}, \quad \gamma=\left(\gamma_{1}, \gamma_{2}\right) \in \mathbb{Z}^{\times 2}
$$

The subset of $T_{\gamma}$ with $\gamma_{1}, \gamma_{2}=0, \ldots, N-1$ is the basis in $\operatorname{Mat}(N, \mathbb{C})$. From (A.16) we have

$$
T_{\alpha} T_{\beta}=\kappa_{\alpha, \beta} T_{\alpha+\beta}, \quad \kappa_{\alpha, \beta}=\exp \left(\frac{\pi \imath}{N}\left(\beta_{1} \alpha_{2}-\beta_{2} \alpha_{1}\right)\right),
$$


where $\alpha+\beta=\left(\alpha_{1}+\beta_{1}, \alpha_{2}+\beta_{2}\right)$. Obviously,

$$
\kappa_{\alpha, \beta} \kappa_{\beta, \alpha}=1, \quad \kappa_{\alpha,-\beta}=\kappa_{-\alpha, \beta}=\kappa_{\beta, \alpha}, \quad \kappa_{\alpha, \beta}=\kappa_{\alpha+\beta, \beta}=\kappa_{\alpha, \beta+\alpha} .
$$

Notice also that

$$
\sum_{\alpha} \kappa_{\alpha, \gamma}^{2}=N^{2} \delta_{\gamma, 0}
$$

where $0=(0,0)$.

For $N=2$ the basis (A.17) in $2 \times 2$ matrices coincides (up to signs) with the Pauli matrices basis $\sigma_{j}$ endowed with $\sigma_{0}=1_{2 \times 2}$. In this case (A.18) can be re-written as $\sigma_{j} \sigma_{k}=\sigma_{0} \delta_{j k}+\imath \varepsilon_{j k n} \sigma_{n}$ together with $\sigma_{j} \sigma_{0}=\sigma_{0} \sigma_{j}=\sigma_{j}$ and $\sigma_{0}^{2}=\sigma_{0}$.

$R$-matrix is defined as

$$
R_{12}^{\hbar}(u)=\sum_{\alpha \in \mathbb{Z}_{N} \times \mathbb{Z}_{N}} \varphi_{\alpha}\left(u, \omega_{\alpha}+\hbar\right) T_{\alpha} \otimes T_{-\alpha} \in \operatorname{Mat}(N, \mathbb{C})^{\otimes 2}
$$

where

$$
\varphi_{\alpha}\left(u, \omega_{\alpha}+\hbar\right)=\exp \left(2 \pi \imath \frac{\alpha_{2}}{N} u\right) \phi\left(u, \omega_{\alpha}+\hbar\right), \quad \omega_{\alpha}=\frac{\alpha_{1}+\alpha_{2} \tau}{N} .
$$

Writing $R_{a b}^{\hbar}(z)$ we mean that $T_{\alpha} \otimes T_{-\alpha}$ in $\left(\right.$ A.21) is replaces by $1 \otimes \ldots 1 \otimes T_{\alpha} \otimes 1 \ldots 1 \otimes T_{-\alpha} \otimes 1 \ldots \otimes 1$ in such a way that $T_{\alpha}$ and $T_{-\alpha}$ acts in the $a$-th and $b$-th components of $\operatorname{Mat}(N, \mathbb{C})^{\otimes n \geq 3}$. If the number of components equals $n$ then $R_{a b}^{\hbar} \in \operatorname{Mat}\left(N^{n}, \mathbb{C}\right)$. With the above definition we get the correct relation

$$
R_{b a}^{\hbar}(z)=P_{a b} R_{a b}^{\hbar}(-z) P_{a b},
$$

where $P_{12}$ is the permutation operator in $\operatorname{Mat}(N, \mathbb{C})^{\otimes 2}$. Notice also that $P_{12}$ has the following form in the basis (A.17):

$$
\sum_{\alpha} T_{\alpha} \otimes T_{-\alpha}=N P_{12}
$$

The classical limit $\hbar \rightarrow 0$ is defined as

$$
R_{12}^{\hbar}(z)=\hbar^{-1} 1 \otimes 1+r_{12}(z)+\hbar m_{12}(z)+O\left(\hbar^{2}\right) .
$$

Then the quantum Yang-Baxter equation (1.3) provides the classical one

$$
\left[r_{a b}, r_{a c}\right]+\left[r_{a c}, r_{b c}\right]+\left[r_{a b}, r_{b c}\right]=0, r_{a b}=r_{a b}\left(z_{a}-z_{b}\right)
$$

Condition (1.7) leads to

$$
r_{a b}=-r_{b a}, \quad m_{a b}=m_{b a}
$$

while (1.8) gives expresses $m_{a b}$ in terms of $r_{a b}$ :

$$
2 m_{12}(z)=r_{12}^{2}(z)-1 \otimes 1 N^{2} \wp(z) .
$$

The answer is similar to $\hbar^{1}$ term in the local expansion of the Kronecker function near $\hbar=0$ :

$$
\phi(\hbar, z)=\hbar^{-1}+E_{1}(z)+\hbar\left(E_{1}^{2}(z)-\wp(z)\right) / 2+O\left(\hbar^{2}\right) .
$$

\footnotetext{
${ }^{6}$ Let us mention that original definition [5] differs from (A.21). It was defined in terms of theta functions with characteristics $1 / N$ and normalized as $R_{12}^{\hbar} R_{21}^{\hbar}=1 \otimes 1$. Its representation in the $\operatorname{standard}$ basis of Mat $(N, \mathbb{C})$ $\left\{\left(E_{i j}\right)_{k l}=\delta_{i k} \delta_{j l}\right\}$ was suggested in [19].
} 
At the same time the Fay identity for $R$-matrix (1.2) gives

$$
r_{a b} r_{a c}-r_{b c} r_{a b}+r_{a c} r_{b c}=m_{a b}+m_{b c}+m_{a c}
$$

Plugging $m_{a b}$ from (A.28) into (A.29) one gets the non-abelian analogue of

$$
\left(E_{1}\left(z_{a}-z_{b}\right)+E_{1}\left(z_{b}-z_{c}\right)+E_{1}\left(z_{c}-z_{a}\right)\right)^{2}=\wp\left(z_{a}-z_{b}\right)+\wp\left(z_{b}-z_{c}\right)+\wp\left(z_{c}-z_{a}\right) .
$$

In the end let us prove the unitarity property (1.8) (this proof was skipped in [13, 14]).

Unitarity. Proof of (1.8). It is convenient to rewrite (1.8) using (1.7) as

$$
R_{12}^{\hbar}(z) R_{12}^{-\hbar}(z)=-N^{2}(\wp(N \hbar)-\wp(z)) 1 \otimes 1 .
$$

The proof is achieved by direct calculation:

$$
R_{12}^{\hbar}(z) R_{12}^{-\hbar}(z)=\sum_{\alpha, \beta} T_{\alpha} T_{\beta} \otimes T_{-\alpha} T_{-\beta} \varphi_{\alpha}\left(z, \omega_{\alpha}+\hbar\right) \varphi_{\beta}\left(z, \omega_{\beta}-\hbar\right)=\sum_{\alpha+\beta=0}+\sum_{\alpha+\beta \neq 0}
$$

The first term reproduces the answer due to (A.7) and $\sum_{\alpha} \wp\left(\omega_{\alpha}+\hbar\right)=N^{2} \wp(N \hbar)$. So we need to prove that the second term (where $\alpha+\beta \neq 0$ ) equals 0 :

$$
\sum_{\alpha+\beta \neq 0} \stackrel{A .18, A .6}{=} \sum_{\gamma \neq 0} \sum_{\alpha+\beta=\gamma} T_{\gamma} \otimes T_{-\gamma} \kappa_{\alpha, \beta}^{2} \varphi_{\gamma}\left(z, \omega_{\gamma}\right)\left(E_{1}(z)+E_{1}\left(\omega_{\alpha}+\hbar\right)+E_{1}\left(\omega_{\beta}-\hbar\right)-E_{1}\left(z+\omega_{\gamma}\right)\right)
$$

The first and the last terms in the brackets are independent of $\alpha, \beta$. Each of them gives 0 since $\kappa_{\alpha, \beta}=\kappa_{\alpha, \alpha+\beta}=\kappa_{\alpha, \gamma}$ and $\sum_{\alpha} \kappa_{\alpha, \gamma}^{2}=N^{2} \delta_{\gamma, 0}$ but the sum does not contain $\gamma=0$ term. Finally, we are left with

$$
\sum_{\alpha+\beta=\gamma} \kappa_{\alpha, \beta}^{2}\left(E_{1}\left(\omega_{\alpha}+\hbar\right)+E_{1}\left(\omega_{\beta}-\hbar\right)\right)=\sum_{\alpha} \kappa_{\alpha, \gamma}^{2}\left(E_{1}\left(\omega_{\alpha}+\hbar\right)+E_{1}\left(\omega_{\gamma}-\omega_{\alpha}-\hbar\right)\right)=0
$$

because the summation index of the second term can be shifted as $\alpha \rightarrow \alpha+\gamma$, and due to $E_{1}(x)=-E_{1}(-x)$.

More details and properties can be found in [19, 12] and [14].

\section{3 $\quad \mathrm{GL}_{N}$ Sklyanin algebra}

The Sklyanin algebra [21] has extension to $\mathrm{GL}_{N}$. One possible approach is given in [17]. The most natural way is to use the exchange relations (as in [21]).

Proposition 5.1 Consider the Lax operator (3.2) and $R$-matrix (1.6). The quantum exchange relations

$$
R_{12}^{\hbar}(z-w) \hat{L}_{1}^{\hbar}(z) \hat{L}_{2}^{\hbar}(w)=\hat{L}_{2}^{\hbar}(w) \hat{L}_{1}^{\hbar}(z) R_{12}^{\hbar}(z-w)
$$

are equivalent to $N^{2} \times N^{2}$ relations numbered by $\alpha, \beta \in \mathbb{Z}_{N}^{\times 2}$ (for the component $T_{\alpha} \otimes T_{\beta}$ ):

$$
\sum_{\gamma} \kappa_{\gamma, \alpha-\beta} \hat{S}_{\alpha-\gamma} \hat{S}_{\beta+\gamma} \mathrm{f}_{\alpha, \beta, \gamma}^{\hbar}=0,
$$

where the structure constants $\mathrm{f}_{\alpha, \beta, \gamma}^{\hbar}$ are given by

$$
\begin{array}{ll}
\text { for } \beta \neq 0: & \mathrm{f}_{\alpha, \beta, \gamma}^{\hbar}=E_{1}\left(\omega_{\gamma}+\hbar\right)-E_{1}\left(\omega_{\alpha-\beta-\gamma}+\hbar\right)+E_{1}\left(\omega_{\alpha-\gamma}+\hbar\right)-E_{1}\left(\omega_{\beta+\gamma}+\hbar\right), \\
\text { for } \beta=0: & \mathrm{f}_{\alpha, 0, \gamma}^{\hbar}=\wp\left(\omega_{\gamma}+\hbar\right)-\wp\left(\omega_{\alpha-\gamma}+\hbar\right) .
\end{array}
$$


For $N=2$ relations (A.30) reproduce $\mathrm{GL}_{2}$ Sklyanin algebra in its original form [21]. Indeed, for $\alpha, \beta \neq 0$ and $\alpha \neq \beta$ the structure constants are related: $\mathrm{f}_{\alpha, \beta, \alpha-\beta}^{\hbar}=\mathrm{f}_{\alpha, \beta,-\beta}^{\hbar}=-\mathrm{f}_{\alpha, \beta, 0}^{\hbar}$ and $\mathrm{f}_{\alpha, \beta, \alpha}^{\hbar}=\mathrm{f}_{\alpha, \beta, 0}^{\hbar}$. Therefore,

$$
\left[\hat{S}_{\alpha}, \hat{S}_{\beta}\right]_{-}=\kappa_{\alpha, \beta}\left[\hat{S}_{\alpha+\beta}, \hat{S}_{0}\right]_{+}
$$

Here we also used that for $\alpha, \beta \neq 0$ and $\alpha \neq \beta \kappa_{\alpha, \beta}= \pm \imath$, i.e. $\kappa_{\alpha, \beta}=-\kappa_{\beta, \alpha}$. To get the second commutation relation introduce

$$
\wp_{\alpha}^{\hbar}=\wp\left(\hbar+\omega_{\alpha}\right)-\wp(\hbar)
$$

and notice that $\mathrm{f}_{\alpha, 0, \alpha}^{\hbar}=-\mathrm{f}_{\alpha, 0,0}^{\hbar}, \mathrm{f}_{\alpha, 0, \beta}^{\hbar}=-\mathrm{f}_{\alpha, 0, \alpha-\beta}^{\hbar}$. Then

$$
\wp_{\alpha}^{\hbar}\left[\hat{S}_{0}, \hat{S}_{\alpha+\beta}\right]_{-}=-\kappa_{\alpha, \beta}\left(\wp_{\alpha}^{\hbar}-\wp_{\beta}^{\hbar}\right)\left[\hat{S}_{\alpha}, \hat{S}_{\beta}\right]_{+} .
$$

for some $\beta \neq \alpha, 0$. Notice also that the same commutation relation appears from $\alpha=0, \beta \neq 0$ component of (A.30) but in a different form:

$$
K_{\alpha+\beta}^{\hbar}\left[\hat{S}_{0}, \hat{S}_{\alpha+\beta}\right]_{-}=\kappa_{\alpha, \beta}\left(K_{\alpha}^{\hbar}-K_{\beta}^{\hbar}\right)\left[\hat{S}_{\alpha}, \hat{S}_{\beta}\right]_{+}
$$

where $K_{\alpha}^{\hbar}=E_{1}\left(\hbar+\omega_{\alpha}\right)-E_{1}(\hbar)-E_{1}\left(\omega_{\alpha}\right)$. The latter is the same as (A.34) due to

$$
\frac{K_{\alpha}^{\hbar}-K_{\beta}^{\hbar}}{K_{\alpha+\beta}^{\hbar}}=-\frac{\wp_{\alpha}^{\hbar}-\wp_{\beta}^{\hbar}}{\wp_{\alpha+\beta}^{\hbar}}
$$

in $\mathrm{GL}_{2}$ case.

\section{References}

[1] M. Aguiar, Contemporary Mathematics 267 (2000) 1-29.

[2] A. Antonov, K. Hasegawa, A. Zabrodin, Nucl. Phys. B 503 (1997) 747-770; arXiv:hep-th/9704074.

[3] J. Avan, E. Ragoucy, V. Rubtsov, arXiv:1401.7629 [math-ph].

[4] R.J. Baxter, Ann. Phys. 70 (1972) 193-228.

[5] A.A. Belavin, Nucl. Phys. B, 180 (1981) 189-200.

[6] I. Burban, B. Kreussler, Memoirs of the Amer. Math. Soc., 220 (2012) 1035; arXiv:0708.1685 [math.AG].

I. Burban, T. Henrich, Journal of the European Mathematical Society, 17:3 (2015) 591-644; arXiv:1202.5738 [math.AG].

T. Henrich, Journal of Physics: Conference Series 346 (2012) 012007.

[7] I.V. Cherednik, Theor. Math. Phys., 43:1 (1980) 356-358.

[8] Yu. Chernyakov, A. Levin, M. Olshanetsky, A. Zotov, J. Phys. A: Math. Gen. 39 (2006) 12083.

[9] D. Chicherin, S.E. Derkachov, V.P. Spiridonov, arXiv:1412.3383 [math-ph].

[10] L.D. Faddeev, L.A. Takhtajan, N.Y. Reshetikhin, Leningrad Math. J. 1 (1990) 193-225.

M. Jimbo, Int. J. Mod. Phys. A 04 (1989) 3759

A. Isaev, Physics of Particles and Nuclei, 26:5 (1995) 501-526.

[11] L. Freidel, J.M. Maillet, Phys. Lett. B 262:2-3 (1991) 278-284. 
[12] K. Hasegawa, J. Math. Phys. 35:11 (1994) 6158-6171.

[13] A. Levin, M. Olshanetsky, A. Zotov, JHEP 10 (2014) 109; arXiv:1408.6246 [hep-th].

[14] A.M. Levin, M.A. Olshanetsky, A.V. Zotov, Theoret. and Math. Phys. 184:1 (2015) 924-939; Teor. Mat Fiz. 184:1 (2015) 41-56; arXiv:1501.07351 [math-ph].

[15] A. Levin, M. Olshanetsky, A. Zotov, JHEP 07 (2014) 012; arXiv:1405.7523 [hep-th].

A. Levin, M. Olshanetsky, A. Zotov, Nuclear Physics B, 887 (2014) 400-422; arXiv:1406.2995 [math-ph].

[16] A. Levin, M. Olshanetsky, A. Smirnov, A. Zotov, J. Phys. A: Math. Theor. 46:3 (2013) 035201; arXiv:1208.5750 [math-ph].

[17] A.V. Odesskii, B.L. Feigin, Funct. Anal. Appl., 23 (1989) 207-214.

[18] A. Polishchuk, Advances in Mathematics 168:1 (2002) 56-95.

[19] M.P. Richey, C.A. Tracy, J. Stat. Phys. 42 (1986) 311-348.

[20] A. Odesskii, V. Rubtsov, V. Sokolov, Int. J. Geom. Methods Mod. Phys. 11 (2014) 1460036; arXiv:1311.4321 [math-ph].

[21] E.K. Sklyanin, Funct. Anal. Appl., 16 (4) (1982) 263-270.

E.K. Sklyanin, Funct. Anal. Appl., 17 (4) (1983) 273-284.

[22] A. Smirnov, Cent. Eur. J. Phys. 8 (4) (2010) 542-554; arXiv:0903.1466 [math-ph].

[23] C.N. Yang, Phys. Rev. Lett. 19 (1967) 1312-1315. 